\title{
23 How Can Brains in Vats Experience a Spatial World? A Puzzle for Internalists
}

\author{
Adam Pautz
}

A "tricked" brain in a vat with exactly the brain activation that I now have would have the same experiences that I am having now despite lacking a body.

-Ned Block

Internalist theories of experience hold that the character of an individual's experiences is entirely fixed by his intrinsic properties, so that any intrinsic duplicate (even a brain in a vat) must have exactly the same experiences. Proponents include Block, Chalmers, Horgan, Kriegel, McLaughlin, Mendelovici, Papineau, and many others. Neuroscientists also typically favor internalism, for instance, Koch, Tononi, Zeki, and Lamme. One version is type-type identity theory: every distinct experiential property is necessarily identical with a distinct intrinsic neurocomputational property of the brain. On the other side are various externalist theories of experience. Examples include tracking representationalism (Dretske, Tye), naïve realism (Campbell, Martin), and active externalism (Noë, O'Regan).

I think that there is a strong empirical case to be made for internalism about experience and against externalist rivals. However, in this chapter my primary aim is not to argue for internalism but to develop an overlooked puzzle for it, a puzzle about the experience of space. My focus throughout is on the type-type identity theory (identity theory for short), which is defended by Block (2009), McLaughlin (2003), Papineau (2014), and others. I focus on the identity theory because it is simple and because there has been some renewed interest in it. I also suggest an answer to the puzzle, an answer

Earlier versions of this chapter were presented at the University of California at Berkeley (2011), Oxford University (2013), the University of Southern California (2014), and Brown University (2014). I thank the audiences on those occasions for very helpful discussions. I would also like to thank Brian Cutter, Uriah Kriegel, Angela Mendelovici, David Papineau, Jeff Speaks, and Daniel Stoljar for helpful comments or discussion. Finally, I am conscious of a considerable philosophical debt to Ned Block, which I hope is evident in these pages. 
having important consequences for our understanding of mental representation and of the place of the mind in the physical world.

Let me give you a sense of the puzzle. Suppose you have an experience that is in fact caused by a round tomato on an all-white background. I think that this experience is necessarily an experience as of a round item of some sort. In the terminology of Chalmers (2004), having it necessarily involves "phenomenally representing" roundness. Now, the puzzle is that identity theorists must apparently say that this representational relation is irreducible. To see this, consider a lifelong, lone brain in a vat (BIV) that formed by chance and that happens to undergo the same brain state as you. On the identity theory, BIV has the very same tomato-like experience as you and so also "phenomenally represents" roundness, despite its degenerate situation. In fact, given the identity theory, BIV might have all your rich visual experiences, representing a range of spatial properties and relations, such as moving to the left, having so-and-so orientation, and being above. In that sense, BIV could ostensibly experience a rich spatial world. It is "tricked" because there aren't things before it with these properties. Obviously, these clusters of properties are also not instantiated inside the brain by the neural states responsible for experience. The puzzle now is roughly this: How can experience be internally determined and yet necessarily externally directed? How can BIV represent properties that are instantiated outside the head (if they are instantiated at all) just on the basis of states inside the head? Evidently, BIV bears no interesting physical-functional relations to such spatial properties, including the relations invoked in our most sophisticated naturalistic theories of representation (asymmetric dependence, indication, and so on). So if, like you, BIV phenomenally represents such properties, then it appears that the phenomenal representation relation must be irreducible: it cannot be identified with any physical-functional relation. But this appears mysterious. ${ }^{1}$

In response to the puzzle, I think internalists should simply concede the irreducibility of the phenomenal representation relation. I think internalists who also subscribe to the doctrine of physicalism should accept what I call the internal grounding view of phenomenal representation. On this view, even though the phenomenal representation relation is irreducible, our bearing this relation to certain shapes and other properties is always "grounded in" (in the sense of Fine, Rosen, and others) our being in certain brain states. I think that this is a defensible view. In fact, it meshes nicely with a general view of representation relations that Paul Horwich and Stephen Schiffer have proposed on independent grounds.

My plan is as follows. In section 1, I explain why we should take internalism seriously. In sections $2-5$, I argue that internalism leads to antireductionism about phenomenal representation. Finally, in section 6, I describe the internal grounding view of phenomenal representation. 


\section{Why Take Internalism about Experience Seriously?}

You will not be very interested in my puzzle for internalism if you are convinced that internalism about experience has no chance of being true. So I first discuss the internalism-externalism debate and explain why I think internalism deserves to be taken seriously. I first explain what I consider to be the strongest argument for the rival position of externalism about experience. Then I show there is a stronger empirical case to be made for internalism and explain why the argument for externalism is not decisive.

The most discussed argument for externalism about experience and against internalism depends on the controversial transparency thesis: whenever you know what your experience is like (even in hallucination), it is by attending to the objects and properties presented in the experience. I think that nonveridical experiences create problems for this thesis (Pautz 2007; see Tye 2014 for a response). I sketch here what I consider to be a stronger argument for externalism about experience, one with a more modest starting point. The argument is that externalists can provide the best explanation of the experience of space. I call it the spatial argument. It helps set the stage for the rest of the chapter. In effect, my puzzle for internalists is about how they might answer this argument.

First, some terminology. Suppose again you see a tomato on a white background. Now pretend that later neuroscientists artificially reproduce exactly the same neural state you now have, so that you have a hallucination that perfectly matches your original experience. Intuitively, you have the same salient mental property in each case. Let $R$ rigidly designate this property. Philosophers would call it the property of having an experience with specific phenomenal character $K$. This is an example of what I call an experience property. Internalism about experience implies that an individual's having $R$ is entirely fixed by the intrinsic properties of his brain, so that even a lifelong BIV could have $R$. Could this be right?

The spatial argument against this internalist view starts with a simple observation. Roughly, you couldn't have $R$ and fail to have an experience as of $a$ round item of some sort. $R$ is necessarily directed at a round item, a kind of item that needn't exist in your brain when you have $R$. Call this External Directedness. Many philosophers have argued for the point. I say more about it later because it figures in my puzzle for internalists about the BIV. For now, let's just assume it. I also assume that having an experience as of a round item is a representational property in some good sense: you can have an experience as of a round item, even if there is no existing round item there. So I put External Directedness by saying that having $R$ necessarily involves phenomenally representing roundness. ${ }^{2}$

The next step in the spatial argument against internalism concerns the following question: Assuming the physicalist view that the physical facts fix all the facts, what 
is the physical basis of your phenomenally representing roundness? For instance, how can you have an experience of a round thing even in hallucination, when there is no physical (or mental) round thing around?

Internalists about $R$ are committed to the following answer: that your intrinsic physical state, considered in isolation from your environment, necessitates your phenomenally representing roundness, a property that is not instantiated by that physical state. For they claim that some intrinsic physical state of you necessitates your having tomato-like experience $R$. And given External Directedness, this in turn necessitates your phenomenally representing roundness.

However, as I indicated at the beginning, this internalist view of the built-in spatial intentionality of visual experience faces an apparent puzzle, which is the main subject of this chapter. In short, the puzzle is that internalism apparently requires irreducible representational relations. In addition, it apparently requires somewhat arbitrary modal connections. For why should simply undergoing a mere neural pattern necessarily result in phenomenally representing roundness and not some other shape, even in possible cases (like the BIV case) in which the brain state is not causally connected to round objects? Considering the intrinsic character of the neural pattern alone, this looks arbitrary.

The best argument for externalism about the experience property $R$, I think, is that it follows from an apparently better externalist account of the spatial intentionality inherent in $R$, one avoiding these puzzles. Since Galileo there has been a question about whether colors-as-we-see-them are really there. But when it comes to the spatial properties and relations we phenomenally represent in experience, nearly everyone accepts realism: these really are occasionally instantiated out there in the world. (We soon see that David Chalmers is an exception.) So an externalist account of how we represent spatial properties is natural: we phenomenally represent spatial properties by having a visual system that interacts with instances of these very properties in the external world.

One simple version is the "tracking" account of Dretske (1995) and Tye (1995). Very roughly, on this account, the physical ground of your representing roundness (and not some other shape) isn't just your undergoing a certain neural pattern alone (as on internalism) but your undergoing a neural pattern that in normal conditions is caused by the presence of a round object (and that in turn causes behavior appropriate to such an object). For short, the ground is your tracking roundness. Call this a tracking property.

This externalist view of spatial experience apparently avoids the kind of puzzle facing the internalist view. It runs no risk of requiring irreducible representational relations: on this view the phenomenal representation relation that links the mind to external spatial properties is just a nonmysterious tracking relation. The externalist view also minimizes arbitrariness. On this view, the physical basis of phenomenally representing roundness is not merely undergoing a neural pattern but undergoing a neural pattern that tracks the instantiation of that very property, roundness. By extending the 
physical substrate beyond the brain, we obtain an explanation of why you have an experience of one shape rather than another.

Now we can complete the spatial argument for externalism about $R$. The starting point was the modest claim that having tomato-like experience $R$ necessarily involves phenomenally representing roundness. The next step was that phenomenally representing roundness depends on more than your intrinsic state: it depends on tracking round things. It follows that having experience property $R$ depends on more than your intrinsic state, just as externalists maintain. For instance, contrary to internalism, this approach entails that an isolated, lifelong BIV simply could not have $R$, even if it is an intrinsic duplicate of your brain, because it doesn't suitably track round things (more on this in section 5).

The spatial argument is general. The character of visual experience is inseparable from representing spatial properties, like moving to the left, having so-and-so orientation, and being above. And in general (so the argument goes) we must explain the representation of such spatial properties in terms of links with the instantiation of these very properties in the external world rather than in terms of mere internal neural patterns considered in isolation from that world. So visual experience properties are not intrinsic.

So far I have focused on the experience of spatial properties, examples of traditional "primary qualities." But what about the experience of traditional "secondary qualities"?

For instance, intuitively, having tomato-like experience $R$ necessarily involves having an experience as of a certain distinctive quality along with roundness. I call it sensible redness. (I call it "sensible redness," rather than just "redness," to remain neutral between the view that it is identical with "the color red" and the rival view that we should rather think of it as a "color-appearance property" that corresponds with but is not identical with the color red.) As Berkeley (1713, 157-158) noted, in general, sensible colors and shapes "appear as being in the same place" (though he himself located them both "in the mind"). Other examples of sensible properties are qualities of sound, bodily pain and pleasure, heat and cold, taste, and so on. We undeniably seem to experience sensible properties as coinstantiated with spatial properties, such as location and shape. Indeed, this is even so in hallucination. How is that possible?

I think that those who accept an externalist account of the experience of primary qualities like shapes might use a generalization argument to support a similar externalist account of the experience of secondary qualities. So the spatial argument indirectly supports externalism generally. This generalization move is supported by considerations of uniformity. On the resulting view, sensible redness, like roundness, is an objective, mind-independent feature of tomatoes and other objects that is tracked by the visual system. In one natural version, it is a reflectance property of objects. Further, we phenomenally represent sensible redness (have experiences of it) in the same way we represent roundness-namely, by having a brain state that tracks it under biologically normal 
conditions. ${ }^{3}$ This theory explains how, even in hallucination, we can ostensibly experience sensible colors in various locations and as conjoined with other spatial properties. Unless we are willing to accept sense-data in a private mental space or the mysterious "visual field regions" of Peacocke (2008), how else might we explain this? True, there are traditional arguments against the view that sensible colors are objective properties of external objects, concerning perceptual variation, spectrum inversion, and so on. But externalists (Dretske, Tye, others) have tried to answer those arguments.

The spatial argument and the generalization argument together suggest that experience properties like $R$ are necessarily connected with tracking properties. If this is right, it is natural to go further and claim that they are just identical with tracking properties. So, for instance, having the tomato-like visual experience $R$ just is having a "suitable" internal state that would, under biologically normal conditions, track the coinstantiation of roundness and a certain sensible color (identified with a reflectance property of surfaces) in a certain viewer-relative place $p$. In general, different experiences (auditory experience, bodily sensations, taste experiences) involve tracking, and thereby representing, different clusters of external properties.

Thus, we have arrived at a general externalist view of experience. In fact, we have arrived at the "tracking representationalism" of Dretske (1995) and Tye (1995). It is "representationalist" in that it holds that phenomenal differences among individuals' experiences reside in differences in what perceptible properties those individuals phenomenally represent. True, there are difficult cases for tracking representationalism (blur, affective differences, attentional differences, itches and tickles), but proponents hope that all, or nearly all, aspects of experience can be handled in this way. For reasons I don't go into here, I consider it to be the best form of externalism.

Tracking representationalism is radically externalist. To see this, it may be helpful to compare it with an internalist theory of experience. Consider, for instance, the brainbased identity theory. To a first approximation, on the identity theory, each experience property is necessarily identical with a unique internal neurocomputational property (e.g., a unique spatiotemporal pattern of neuronal firing) rather than with a tracking property involving the external world. (I provide a more complete formulation in section 2.) Differences among experiences are constituted by differences in these patterns (see Prinz 2012, 126-133, for a very important discussion). If you want to know the complete essence of having a reddish experience, you would have to look at the corresponding spatiotemporal neural pattern. True, as a matter of fact, a reflectance property of external objects normally causes this neural pattern; but the experience has no essential connection to that reflectance property. Likewise, the essence of the smell of peppermint is another (and presumably radically different) internal spatiotemporal neural pattern. By contrast, on the tracking representationalism of Dretske and Tye, experience properties are identical with tracking properties of the following form: having an appropriate internal state that tracks clusters of spatial and other external properties 
$P, Q, R$, and so on. Differences between experiences (within a species or across species) reside wholly in differences in the external physical properties normally tracked, and thereby represented, by those experiences. So if you want to know the essence of a reddish experience of a tomato, look at the reflectance property that it tracks (constituting the sensible redness you perceive, on this view) —not the neural "content vehicle." And if you want to know the essence of a smell experience, look at the chemical property that it tracks. As Tye says, "Peer as long as you like at the detailed functioning of the brain ... that is not where phenomenal character is to be found" $(1995,162-163)$. Elsewhere he explains, "Phenomenal character is in the world" $(2009,119)$.

So far, I have sketched a seductive spatial argument for externalism about experience, which led us to the "tracking representationalism" of Dretske and Tye. Let us now turn to the argument for the other side, internalism about experience.

There are many arguments against externalist views like tracking representationalism and for internalism about experience. Many of them are armchair arguments. In my view, such arguments are unconvincing (Pautz 2013b). For instance, the inverted spectrum argument says that it is conceivable, and therefore possible, that two people should have "inverted color experiences" but normally track the same reflectance properties in the external world (Block and Fodor 1972; Shoemaker 1994). But the move from conceivability to possibility is questionable (Tye 2000, 109-110). Block's (1994) well-known Inverted Earth argument-which is the flip side of the inverted spectrum argumentcan be shown to depend crucially on the assumption of internalism about experience (Levine 2001, 113). In his formulation of the argument, Block asserts without argument that "we can assume the supervenience of qualia on the brain" $(1994,518)$. But this is exactly what is at issue. We need an argument for this assumption.

In my view, the best argument against externalism and for internalism is an empirical argument, which I call internal-dependence argument. I recommend that internalists add this argument to their arsenal. To illustrate the internal-dependence argument, I focus on the identity theory, but I think a similar argument could be developed for other internalist theories. Elsewhere I have explained the argument in detail, and have distinguished it from more standard arguments. ${ }^{4}$ Here I can only briefly describe some of the recent empirical work it is based on. Much of this work uses the emerging technique of multivariate pattern analysis. While the spatial argument for externalism starts with our experience of spatial properties (traditional primary qualities), the internal dependence for internalism starts with the experience of sensible properties (secondary qualities).

For instance, suppose you successively experience blue, purple, and green. Then your first color experience is more like your second than your third. On tracking representationalism, all facts about the character of our experiences derive from fact about the physical properties tracked and thereby represented by our experiences. But it is simply not the case that the "blue" reflectance-type is more like the "purple" reflectance-type 
than the "green" reflectance-type, where these are the reflectance-types (colors) tracked and thereby represented by your consecutive experiences. In fact, if anything, the blue reflectance-type is more like the green one than the purple one (Byrne and Hilbert 2003; Pautz 2006b).

At the same time, important recent research in neuroscience shows that phenomenal similarities and differences map nicely onto neural similarities and differences. Brouwer and Heeger sum up this research as follows:

The visual system encodes color by means of a distributed [neural] representation [in area V4].... Similar colors evoke similar patterns of [neural] activity, and neural representations of color [in V4] can be characterized by low-dimensional "neural color spaces" in which the positions of [experienced] colors capture similarities between corresponding patterns of activity. $(2013,15454)$

Indeed, when ordered according to similarity, these neural representations form a circle, akin to the familiar hue circle. So your distributed internal V4 neural representation of the blue object resembles your V4 neural representation of the purple object more than your V4 neural representation of the green object. Other recent important work on the neural basis of color experience includes Danilova and Mollon (2012) and Schmidt, Neitz, and Neitz (2014). In light of this recent research, a broadly internalist account of color experience is evidently more reasonable than an externalist theory such as tracking representationalism. Elsewhere I have developed this point more precisely by appealing to hypothetical "coincidental variation" cases, which differ from both inverted spectrum and Inverted Earth cases. ${ }^{5}$

The point extends to nonvisual modalities. For instance, phenomenal resemblances among smells radically fail to line up with objective resemblances among the corresponding chemical properties tracked, and thereby represented, by our olfactory system (Pautz 2013a). By contrast, research conducted by Youngentob and colleagues (2006), Howard and colleagues (2009), and others has shown that neural patterns in the olfactory system fall into a neural similarity space nicely matching phenomenal similarity space (similar to Brouwer and Heeger [2009] for color vision). ${ }^{6}$ Similar results have been found for the experience of taste (Crouzet et al. 2015). Finally, psychophysics has shown that there is huge mismatch between the qualitative structure of auditory experiences (e.g., ratio relations among perceived loudness levels, categorical changes in phoneme perception) and the structure of the physical properties our auditory experiences track and thereby represent. To find the explanation, we must look inside the head (Chang et al. 2010; Pautz 2013a). This is exactly the opposite of what tracking representationalists like Dretske and Tye suggest.

In short, in many cases, externalists like Dretske and Tye have it backward. Peer at the external physical properties tracked and represented by our experiences however much you like. That is not where you will find an explanation of the phenomenal 
structure of our experiences. So the explanation must reside in the "detailed functioning of the brain." And the more we learn about the brain, the more this hypothesis is corroborated. My point here is not just that in the actual world experiential differences are always accompanied by neural differences. Contrary to what some have suggested (e.g., Prinz 2012, 19), that fact alone provides no evidence against externalism about experience (Pautz 2013b, 168). My point relies on two additional empirical facts. First, in many cases, psychophysics shows that structural relations among experiences (similarity and difference, equal intervals, proportion) are not matched by the structural relations among the (highly unnatural) external physical properties that those experiences track. Second, at the same time, recent neuroscience has shown (often using multivariate pattern analysis) that they are much better matched by structural relations among their neural correlates. These twin facts suggest that our experiences of sensible properties (sensible colors, smells, audible qualities) depend on neural processing, in a way that can be demonstrated to be in conflict with tracking representationalism (Pautz 2013a).

Once we accept an internalist account of the experience of sensible properties, the simplest and most uniform view is that the experience of all perceptible properties (including spatiotemporal ones) generally is internally determined. The brain-based identity theory is a view of this kind. Like the argument for externalism we consider above, this is a generalization argument-only it proceeds in the opposite direction.

So there is a strong empirical case for internalism about experience. But then what about the more a priori spatial argument against internalism and for externalism that we started with? That argument suggests that spatial phenomenology, at least, does depend constitutively on links to the environment, contrary to a general internalist theory like the identity theory.

In my view, the spatial argument is far from decisive, for a couple of reasons. To begin with, the spatial argument for externalism about experience depends on realism about the spatial properties that we phenomenally represent. The thought is that, since these properties are instantiated out in the external world, it's natural to explain how we phenomenally represent them in terms of our having a visual system that interacts with instances of these very properties in the external world.

But some have recently suggested a kind of irrealism about experienced spatial properties. This view may seem far-fetched, but several philosophers have advocated it on the basis of contemporary physics (for discussion, see Ney 2013, 177-181). One example is David Chalmers. In fact, Chalmers advocates a kind of generalized irrealist view. To illustrate, suppose you view a tomato. Then it seems to you that a certain quality, sensible redness, is coinstantiated with roundness. Since the seventeenth-century scientific revolution, many have suggested that such sensible colors are not really instantiated by external objects. These "irrealists" about sensible color would admit the tomato has a reflectance. But, in their view, this reflectance is nothing like the sensible color 
presented in experience. Chalmers accepts this irrealist view of sensible colors on a priori grounds $(2006,82)$. I have also argued for the same view on entirely empirical grounds (Pautz 2006b, 2013a). What Chalmers does is take irrealism one step further. The "real tomato" does not even instantiate the property being round that you phenomenally represent as coinstantiated with sensible redness-that is, the property having edges roughly equidistant from a common point. Chalmers $(2006,107)$ calls this "perfect roundness"; if you like, it is "roundness-as-we-see-it." On his view, the tomato has only "imperfect roundness," which is some arcane quantum mechanical property that is nothing like the familiar roundness you are directly acquainted with (Chalmers 2012, 296-297). In general, the basic spatial and temporal relations given in experience are just not out there. Chalmers thinks that this irrealist view is supported by relativity and certain interpretations of quantum mechanics ("wavefunction fundamentalism"). The result is a kind of uniform Kantian picture: the real world is "noumenal." ${ }^{7}$ As Chalmers puts it,

In spatial experience, I think we are presented with certain primitive spatial properties.... But I think that there is little reason to think that they are instantiated in our world. Certainly, it is not easy to see how [they could be instantiated] in a relativistic world, or in a string-theoretic world. $(2012,333)$

If Chalmers thinks that "perfect" roundness and redness are not instantiated in the external world, where does he think they are instantiated? Does he perhaps think that they are mental qualia instantiated in the mind or the brain when you experience the tomato? No-of course, when you experience a tomato, perfect roundness (that is, the property having edges roughly equidistant from a common point) need not be instantiated inside your brain! Instead, Chalmers adopts a representationalist view. In having the tomato-like experience, you phenomenally represent perfect roundness and redness. So they appear to be instantiated out there. Chalmers, then, accepts External Directedness where "roundness" is understood to mean perfect roundness. But he thinks that, as it happens, nothing instantiates these properties-not even mental items in the head. It is only in some other possible worlds ("Edenic worlds") that they are instantiated by external objects, according to Chalmers.

Of course, if such an irrealist view of experienced spatial properties is correct, then the spatial argument for externalism fails at the first step. We cannot explain how we phenomenally represent roundness-as-we-see-it (much less redness-as-we-see-it) in terms of being in an internal state that typically tracks the instantiation of that very property under biologically normal conditions, for the simple reason that it could never be instantiated in this world! The irrealist view goes with internalism about the spatial intentionality built into normal visual experiences. ${ }^{8}$

My point here is not that this irrealist view is definitely right. My point is just that it may be correct. So the spatial argument for externalism about visual experience is not so cut and dried. (However, in what follows, for the sake of discussion, I often write 
as if realism about experienced spatial properties is correct.) In fact, although I cannot go into this here, there are other empirical reasons to doubt externalist theories of the experience of space. So it is worthwhile to consider the question of whether we can develop an alternative internalist theory. ${ }^{9}$

There is another, more basic reason why the spatial argument isn't a decisive argument against internalism about experience. That argument is just an inference to the best explanation. The argument is that an externalist theory, such as the tracking theory, provides the best explanation of the spatial intentionality built into many experiences. So one way to block the argument would be to show that there is a viable rival internalist explanation of the experience of spatial features. Such a theory would explain how BIV, just on the basis of its internal neural states, can "phenomenally represent" roundness, even though it fails to track round things.

The rest of this chapter is devoted to the question of what such an internalist theory might look like. I begin by developing the puzzle about spatial representation that is the main focus. In particular, I argue (sections 2-5) that internalists must concede that the "phenomenal representation relation" is an irreducible relation. This may look like a reductio of internalism. However, I briefly suggest (section 6) that this is not the right way to look at it. Internalists can live with this result by accepting a nonreductive grounding account of the phenomenal representation of space.

\section{From Envatted Brains to Irreducible Intentionality: The BIV Argument Sketched}

The BIV argument is designed to establish a conditional claim: if internalism about experience is right, then a nonreductive view of phenomenal representation follows.

In the present section, I describe the setup and list the steps of the argument. Then, in sections 3-5, I explain and defend those steps in turn. I continue to focus on the tomato-like experience property $R$. However, just about any visual experience could illustrate the argument. I also continue to focus on the brain-based identity theory defended by Block, McLaughlin, Papineau, and others.

I begin by saying more about the identity theory. I have said that the identity theory holds that $R$ is necessarily identical with an intrinsic neurocomputational property of the brain ("intrinsic" in the sense that any duplicate brain must share the property). But what kind of property? Suppose that whether a subject has tomato-like experience $R$ covaries with having some fairly local neural property $V$ in the visual cortex. No identity theorist holds that $V$ all by itself-say, isolated in a bottle-would constitute $R$. Rather, identity theorists hold that $R$ is necessarily identical with some more global neural state, incorporating certain further conditions. Let $N$ be the more global neural state that, by contrast to $V$ alone, is necessary and sufficient for $R$, according to identity theorists.

What might $N$ involve? Ned Block (2005, Box 1), one of the most prominent defenders of a brain-based approach to experience, suggests that we can approach the issue by 
asking, What could be removed from your brain, and what must be kept, while you still continue to have $R$ ? Exactly which brain areas are required is unknown at present. But Block tentatively suggests that $N$ probably has to incorporate a "recurrent feedback loop." He also notes that "there is some evidence that there is a single neural background of all experience involving connections between the cortex and the upper brain stem including the thalamus" (Block 2007a, 482).

But we could remove other things. As Block writes, citing research, "The basic phenomenology of vision can survive vast destruction in motor areas and early sensory areas on both sides of the brain" (Block and O'Regan 2012, sec. 10). You could have $R$ because of direct stimulation of your visual cortex, without involving the eyes. In addition, we could remove connections to the body: for, contrary to behaviorists, a quadriplegic, or someone with complete locked-in syndrome, could have $R$. Therefore $N$, the minimal sufficient condition for $R$, doesn't involve the eyes or the motor output systems (e.g., motor neurons in the spinal cord and brainstem).$^{10}$

Now for the setup of the argument. Suppose that, in another possible world, a BIV just pops into existence out of the blue (like a Boltzmann brain), and then starts to undergo neural state $N$ for five minutes purely by chance, without any external cause (Block and O'Regan 2012, sec. 12). Suppose, further, that BIV has only the neural machinery required to have $N$ and hence $R$. BIV doesn't have, for instance, receptor systems (eyes, ears, etc.) or a motor output system. Although I called it a "brain," BIV is not exactly like a complete brain. Indeed, it has no evolutionary history and belongs to no species (in fact, we could assume that BIV occupies an otherwise empty universe, so that it is a brain in the void). So it doesn't really count as a human brain. It is merely an intrinsic duplicate of a significant part of your actual brain as you view a tomato.

Still, the identity theory implies that BIV has the tomato-like experience property $R$. Likewise for any internalist theory. This cannot be disputed. For I have stipulated that BIV has $N$, where " $N$ " is just shorthand for whatever neural property is, on that theory, the minimal neural basis of $R$.

This is not a trivial implication. As we shall see, Dretske and Tye deny it, on the basis of their tracking representationalism. ${ }^{11}$ In what follows, I assume it for the sake of argument.

Here now is an initial, summary statement of the BIV argument:

Assumption. An internalist theory of experience is right, so that, by having neural property $N$, BIV has experience property $R$. (Assume for conditional proof.)

External Directedness about $R$. Necessarily, if any individual has $R$, then that individual has an experience of a round item of some sort; that individual has an experience that "matches the world" only if some item is present that is round. So in having $R$, BIV has such an experience.

Phenomenal representation. If BIV has an experience that "matches the world" only if something is present that is round, then it stands in the following dyadic relation to the property being 
round: it has an experience that "matches the world" only if some item has this property. Call this the "phenomenal representation relation."

Irreducibility. If BIV bears the dyadic phenomenal representation to the property of being round, then this relation is irreducible. For BIV bears no suitable dyadic physical-functional relation to the property of being round, with which the phenomenal representation relation might be identified (e.g., the kind of "tracking relation" invoked by Dretske and Tye).

Conclusion. If an internalist theory of experience is right, so that BIV has $R$, then the dyadic phenomenal representation relation is irreducible.

The conclusion of the BIV argument is depicted in figure 23.1.

An internalist who accepts the BIV argument faces the question of how to understand the relationship between the irreducible phenomenal representation relation and the physical world. This constitutes the puzzle of the phenomenal representation relation for internalists.

Before I turn to elaborating and defending the steps of the BIV argument, let me briefly address an important issue. The puzzle of phenomenal representation is bound up with another puzzle for internalists, which we might call the puzzle of sensible properties. As I noted above, in having tomato-like experience $R$, BIV necessarily has an experience as of a certain distinctive reddish quality filling a round area in space; that is, BIV phenomenally represents "sensible redness" as well as roundness. We saw that externalists like Tye and Dretske have an attractively straightforward (though arguably false) view of sensible redness: sensible redness is an external reflectance property that really is coinstantiated with roundness. But internalists about experience cannot take this

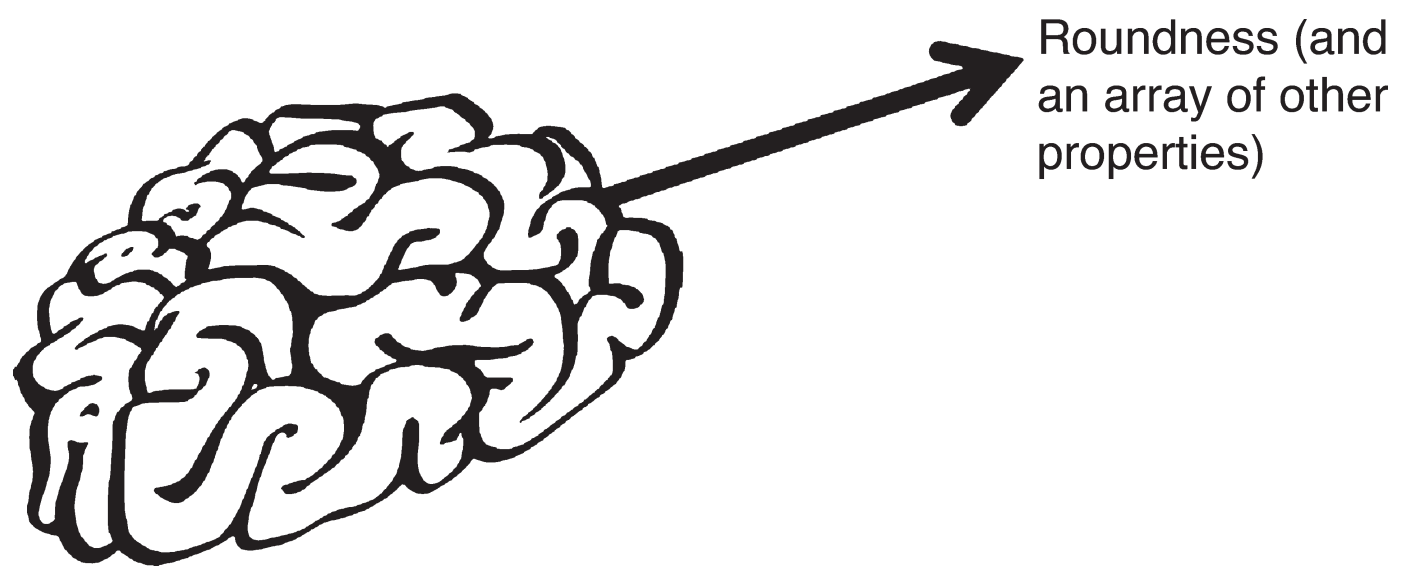

Figure 23.1

Internalism about experience implies that the phenomenal representation is irreducible (represented here by the arrow sticking out from the brain). 
objectivist view. For they hold that internal neural state $N$ suffices for the experience of sensible redness, even in BIV cases in which it doesn't track any reflectance property in the external world. The empirical research on color vision I cite above supports this brain-based view. But then, according to internalists, what in the world might sensible redness be, if not an objective property of external things in physical space?

Ned Block would apparently say that, when BIV (or for that matter, an actual person) has the tomato-like experience, sensible redness (or what he calls the "red quale") is somehow instantiated "in the mind" (2007b, 74). On one version of this view, sensible redness is instantiated by a literally round "visual field region." But there exists no reddish and literally round "visual field region" anywhere within BIV (Peacocke 2008, 14). On Block's own view, sensible redness (the "red quale") is instead instantiated by the BIV's experience itself, which, on his identity theory, is just a neural state $N$. But, necessarily, if anyone has the tomato-like-experience, then it at least seems to him that sensible redness fills a round region; in general, sensible colors appear to fill regions and stand in spatial relations. So Block's view has the odd implication that a quality that does not in fact fill a round region (because it is a quality of a nonround neural state) somehow appears to fill a round region (see Pautz 2013a, 286-290). Sydney Shoemaker (1994) is an internalist who suggests a different view of sensible redness. He suggests that it is a response-dependent "appearance property" (roughly, a disposition to produce a certain neural state in a population) that is really instantiated by external objects (like round tomatoes) in our environment but not in BIV's environment. Other internalists have argued that sensible redness is a primitive property that is not instantiated anywhere, on both a priori grounds (Chalmers 2006; Horgan 2014) and empirical grounds (Pautz 2006b, 2013a).

If internalism is true, then which of these views on the nature and whereabouts of sensible redness is best? And what makes it the case that BIV ostensibly experiences sensible redness as bound with roundness? These questions make up the puzzle of the sensible properties for internalists.

In the following sections, I elaborate and defend the steps of the BIV argument for the irreducibility of phenomenal representation. I mostly ignore the whole issue of the nature of sensible properties like sensible redness. Instead I mostly focus on how the BIV might phenomenally represent spatial properties like roundness. The BIV argument goes through no matter what view of sensible properties the internalist adopts.

\section{First Step: External Directedness}

The first step of the BIV argument is this:

External Directedness: necessarily, if any individual has tomato-like experience $R$, then that individual has an experience as of a round item of some kind. Further, that experience fully matches the world only if there is some item present that is round, that has edges roughly equidistant from a common point. 
I call this "External Directedness" because when our experiences are "directed at" items of various shapes and standing in various spatial relations, such items need not be in our head. I already briefly alluded to External Directedness in the seductive spatial argument for externalism about experience (section 1). Now I clarify and defend it in more detail.

The notion of "matching the world" employed in the formulation of External Directedness can be explained by examples. Suppose you have the tomato-like experience $R$ while hallucinating. Your experience does not match the world if there is really only a rectangular table before you. But if there happens to be before you a tomato on a white background, then we can all recognize a sense in which your hallucination does match the world (many philosophers have discussed such veridical hallucinations).

My BIV argument requires only that the single experience $R$ is necessarily directed at a round item. But I think similar claims apply to other visual experiences: other experience-types might be necessarily directed at a square object, or an object moving from left to right, or one object being above another.

Why accept External Directedness? The initial argument is based on reflection. Consider the sentence "a round thing is present." Since language is conventional, we can easily imagine hypothetical cases in which those very marks mean that a triangular thing is present or mean nothing at all. But could someone have $R$ (an experience exactly like your experience of a stationary tomato on a white background) while not having an experience as of a stationary round thing, a thing whose edges are roughly equidistant from a common point? For instance, could someone have that very experience and yet have an experience that is correctly characterized as an experience as of a triangular thing moving to the right? That just seems impossible. So even when BIV has $R, \mathrm{BIV}$ has an experience as of a round thing. This, together with the absence of a round thing, is the only explanation of the evident fact that BIV has a nonveridical experience. As Block (in Block and O'Regan 2012, sec. 12) puts it, BIV is "tricked."

There are other arguments for External Directedness. For instance, if a BIV with the general capacity for thought had $R$, it would thereby be in a position to have a false (but justified) thought with the content something is that way, a thought that is true only if something is round. Despite its sorry state, it could acquire a demonstrative concept of roundness. In general, having visual experiences is what explains our ability to form concepts of spatial properties and relations. It has a unique explanatory significance. How could this be so if visual experience did not itself have built-in spatial content? (For another important argument for External Directedness, see Chalmers 2006, 74.)

The assertion that some visual experiences are necessarily externally directed is neutral on many questions. For instance, one question is, When you view a tilted penny, is the type of experience you have necessarily as of a thing that is elliptical, or of a thing 
that is tilted and round, or as of a thing that is elliptical from here (the viewpoint-relative but objective property of having a shape that would be occluded by an ellipse placed in a plane perpendicular to the line of sight) $?^{12}$ Or are multiple answers correct? And do the spatial predicates needed to characterize the full accuracy conditions of experience express spatial properties that physical objects sometimes really have (Horgan 2014), or does contemporary physics show that this is not so (Chalmers 2012)? As I develop it, my BIV argument is neutral on these issues.

External Directedness is also neutral between the main theories of experience. For instance, while it is implied by all versions of representationalism (including those of Tye, Dretske, Chalmers, and Horgan), it is also strictly speaking compatible with a purely nonrepresentational view of visual experience, which denies that visual experience is essentially representational. In particular, the internalist might deny that BIV's tomato-like experience $R$ has any representational content at all (perhaps on externalist grounds). Still, the internalist might accommodate External Directedness by invoking the traditional sense-datum theory or Peacocke's sensationalism (2008). On this approach, BIV counts as having an experience "as of a round item," in accordance with the letter of External Directedness, because BIV has an experience of a reddish and literally round visual field region. On this view, the experience matches a scene only if the scene contains an object with the same shape as the visual field region.

But such a "visual field region" would be a peculiar nonphysical item, for there is certainly no such round physical object inside BIV's brain. So internalists should reject this view. They should not recognize in this case the real existence of any such object (Peacocke 2008, 14).

So I think internalists should accept a broadly representational interpretation of External Directedness. BIV's experience simply has a false representational content to the effect of there is a round item right there or that is round.

This still doesn't amount to representationalism about experience. Even on a representational interpretation, External Directedness implies only that in some cases visual experiences are necessarily connected with representational properties, specifically, representational properties involving space. This falls short of the representationalist thesis that, necessarily, all experiential facts consist in facts about the contents of our experiences. So even antirepresentationalists could accept External Directedness.

In fact, Ned Block, a well-known antirepresentationalist, favors External Directedness. He writes,

[Representationalism] is the view that the phenomenology of an experience is the experience's representational (intentional) content. I am an opponent. But I am willing to allow that every phenomenological state has representational content, even that the phenomenology consists—in part-in its having that representational content. (2007a, 538) 
Elsewhere Block $(1995,278)$ explicitly says that some types of visual phenomenology are "intrinsically" tied to certain spatial representational contents (there is a circle there, there is a square there, and so on), in agreement with External Directedness.

External Directedness is a modal claim. It says that $R$ is necessarily directed at a round item. So the case for it is incomplete until we consider whether it holds up in hypothetical cases.

Imagine that, on a Twin Earth, tomatoes are (what we would call) ellipsoid. Nevertheless, suppose that our Twin Earthlings are wired so that they normally have experience $R$ (which we have on viewing round tomatoes) on viewing these ellipsoid tomatoes. Is $R$ in any sense an experience as of a round thing, even on this Twin Earth where it is normally caused by ellipsoid objects? External Directedness implies a yes verdict. Two reasonable accounts of the case are compatible with this verdict. (1) On one account, while we get it right, our counterparts are regularly subject to a mild shape illusion. Horgan's (2014) realism about experienced spatial features implies this verdict. (2) There is also Chalmers's irrealist view, which I described above. On his view, in accordance with External Directedness, $R$ is necessarily directed at a "round" thing (or in his terminology, a "perfectly" round thing) on Twin Earth as well as Earth. But neither tomatoes on Twin Earth nor tomatoes here on Earth possess this property of "perfect roundness" or "roundness-as-we-see-it." Rather, on the different planets, tomatoes have merely different versions of "imperfect" roundness; they have only (different) arcane quantum mechanical properties, neither of which is anything like the "perfect" roundness given in experience (roundness-as-we-see-it). The result is that neither our experience of our tomatoes nor our twins' experience of their tomatoes is perfectly veridical! ${ }^{13}$

Let me address a final issue. You might think identity theorists, and internalists in general, should reject External Directedness, blocking my BIV argument at the first step.

In fact, the BIV case itself can be used to illustrate the thought. On internalism about experience, in having neural state $N$, BIV has the tomato-like experience $R$, despite being isolated from the external world. But assuming a standard externalist approach to perceptual representation (e.g., Burge 2010), even though BIV has $R$ (the very same experience you have on viewing a tomato), BIV cannot count as having an experience as of a round thing, contrary to External Directedness. For in BIV, the neural state $N$ does not have the required biological function of tracking round things or indeed things of any kind. In fact, assuming standard externalism, BIV's highly detailed tomato-like visual experience has no content at all (somewhat like a state of undirected depression). In the BIV scenario, there is simply no sense in which the tomato-like experience $R$ is essentially an experience as of a round item (e.g., no nonphysical round sense-datum or visual field region is present either), even though it is exactly like your experience of a tomato. In this regard, the tomato-like experience $R$ is like the marks "a round thing 
is present": this very experience could have had no spatial content at all or could have had any spatial content you please.

However, I think that internalists about experience (including identity theorists) should accept External Directedness and reject this argument. For there are very strong arguments in favor of External Directedness, as we have seen. (Again, if BIV's tomatolike experience has no content at all, why consider it nonveridical?) Chalmers (2006) and Horgan (2014) are examples of philosophers who combine internalism about experience with External Directedness. As we have seen, Block, too, says that some visual experiences are "intrinsically" linked to certain spatial contents.

To block the above argument against External Directedness, internalists about experience should simply reject the assumption of externalism about phenomenal representation on which it depends. As Chalmers $(2006,83)$ and Horgan (2014) have emphasized, even if externalism is right in some cases (representational states about individuals or natural kinds like tomato), it simply does not follow it is true for all representational properties, including the representational properties of experience. Some basic forms of phenomenal representation must be internally determined, if they are (as Block puts it) "intrinsically" linked with phenomenology and phenomenology is internally determined. So, for instance, by having the tomato-like experience $R$, BIV counts as having an experience as of a round object. This, together with the absence of a round thing, explains why the experience is nonveridical. I don't see how the internalist could plausibly deny this. ${ }^{14}$

\section{Second Step: Phenomenal Representation}

The ultimate aim of my BIV argument is to show that internalism about experience requires a nonreductive theory of phenomenal representation. The first step was External Directedness: if BIV has the tomato-like experience $R$, then BIV thereby has experience as of a round thing, an experience that matches the world only if a round thing is present. The next step is to argue that, if this is right, then BIV bears the "phenomenal representation relation" to the property of being round (as depicted in figure 23.1).

I assume a minimal realism about properties: there exists a rich set of spatial properties in the BIV scenario, instantiated by various objects (objects with which BIV cannot causally interact).

If there are properties, then the claim that BIV has an experience that matches the world only if something is round is equivalent to the claim that BIV has an experience that matches the world only if something has the property of being round. This claim in turn immediately implies that BIV bears a representational relation to the property or attribute of being round. A rough gloss on this relation is as follows: $x \lambda x \lambda y(x$ has an experience that matches the world only if something has property $y$ ). I call this the phenomenal representation relation, following Chalmers (2004). As Burge puts it, experience 
involves the perceptual attribution "of certain types or attributes-such as roundness, being to the left of" $(2010,380)$. (My focus here on the general element of perceptual content is of course consistent with acknowledging a singular element.) In general, if you have the general capacity for thought and if you phenomenally represent a certain property, then you thereby have the capacity to predicate that property of things in thought.

So if identity theorists accept External Directedness, as I have argued they should, then they must claim that, when BIV undergoes neural state $N$ (on this view, visual experience property $R$ ), BIV thereby phenomenally represents roundness. As I have defined it, this is a dyadic relation between subjects and properties. Many internalists already accept this result, for instance, Chalmers $(2006,107)$ and Horgan (2014). It is a very minimal claim that doesn't go beyond the pretheoretical claim of External Directedness. For instance, it doesn't require the additional claim that BIV's experience "aims at the truth" in the way beliefs do, or "lays claim" to the presence of a round thing (Papineau 2016, sec. 15). In fact, on some interpretations, I myself reject this additional claim (Pautz 2010c, 269n11).

The argument generalizes to other spatial and temporal properties. Assuming internalism, BIV might have all the same experiences as you by having the same underlying neural states. Then, like you, BIV stands in the phenomenal representation relation to a large variety of spatial properties and relations, such as having orientation l, being above, and having an edge at viewer relative place $p$. That is to say, it has experiences that match the world on the condition that there are things before BIV having these properties.

True, BIV does not see any instances of these properties. But the general properties still exist, and (by my argument) BIV bears a representational relation to them, in the above sense. In the actual world, whenever an individual has a hallucination, they likewise bear the phenomenal representation relation to perceptible properties that aren't instantiated before them. Indeed, something similar happens whenever you have a false belief. If you mistakenly believe something in the next room is round, then you bear the following relation to being round, even though it is not instantiated in your vicinity: you have a belief that is true on the condition that something in the next room have this property.

Recall that I am assuming that in the BIV scenario all of the relevant properties are instantiated by some objects or other (objects BIV cannot causally interact with). So even internalists who are leery of Platonic, uninstantiated properties-for instance, Mendelovici (2010) and Kriegel (2011)—must admit that these properties exist in the BIV case and that in this case BIV bears the phenomenal representation relation to them. So they too face the question I am leading up to of whether this relation is reducible (section 5). ${ }^{15}$

Of course, BIV phenomenally represents what I (in sections 1 and 2) call sensible colors in addition to spatial properties. For BIV has exactly the same experience $R$ you 
had on viewing a particular tomato. And the following expanded version of External Directedness is hard to deny:

External Directedness II: Necessarily, if an individual has the tomato-like experience $R$, that individual has an experience as of an item that is round and red $_{s}$.

Here the predicate "red," could be defined ostensively: it expresses that familiar, salient property that seems to you and your BIV counterpart to fill a round region when you have the tomato-like experience $R$. (I take it that such properties exist, for we can say true things about them: for instance, that red $_{s}$ is more like orange than green $\mathrm{n}_{\mathrm{s}}$.) As Papineau (2016) puts it, you and your BIV counterpart "encounter" this property. It is undeniable that it appears to BIV that this property is coinstantiated with roundness. One explanation is that it is a property of a literally round "visual field region" (Peacocke 2008), but we should reject such items. We should rather say that the BIV phenomenally represents this sensible property as coinstantiated with roundness (Chalmers 2006; Horgan 2014).

BIV might phenomenally represent various other sensible properties. For instance, if it has an auditory hallucination, it has an experience as of an event having a certain location and certain audible qualities (sensible pitch and loudness). Its auditory experiences fully match the world only if these properties are coinstantiated in a space around it. In that sense, BIV phenomenally represents the conjunction of a certain location and certain audible qualities.

To sum up so far: internalists must hold that, simply in virtue of its neural states, BIV somehow phenomenally represents a large variety of spatial properties and relations as well as certain sensible properties.

\section{Final Step: Irreducibility}

The final step of the BIV argument is that, if BIV bears the phenomenal representation relation to spatial and other properties, then this relation is irreducible.

Before developing the argument, let me briefly explain what I mean by saying that a property or relation is reducible. Roughly, I say that a property $P$ is reducible to properties and relations $Q, R, S, \ldots$ just in case $P$ is identical with a complex property built up from $Q, R, S, \ldots$ (so that $Q, R, S, \ldots$ are "ontologically prior" to $P$ ). ${ }^{16}$ The identity theory that is the focus of my discussion is a reductive theory of monadic experience properties. On this theory, the experience property $R$ just is the complex neural-computational property $N$.

Many advocate reductionism about all manifest image properties: they hold that they are one and all nothing but complex properties built up from some limited set of properties and relations from the scientific image (e.g., the properties of the physical 
sciences plus certain topic-neutral properties and relations causes and is a part of). In this sense, they defend reductive physicalism. They include Armstrong, Field, Jackson, Lewis, Papineau, Sider, and Smart. I myself think that this approach is right for nearly all manifest image properties instantiated in the world (with the phenomenal representation relation being a major exception).

I generally favor reductionism because it provides the simplest explanation of the relation between the manifest image and the scientific image. It is ontologically simple, since it holds that manifest image properties are just identical with properties built up from scientific image properties. It is also simple in its stock of brute principles. It requires only general principles of property construction that everyone accepts. True, it requires brute identities between manifest image properties and complex physicalfunctional properties. But as many have emphasized, identities have a very attractive feature: they do not cry out for further explanation. They are explanation stoppers. And intuitively, identities do not add to the complexity of a theory.

Further, as Sider (2011, chap. 7) has emphasized, standard arguments against reductionism about the manifest image fail. For instance, along with Sider, I use "reduction" broadly enough that functionalist theories are versions of reductionism. So "reductionism" in my sense accommodates multiple realizability (e.g., being a chair might be identical with a functional property). In addition, the history of failed attempts to provide complete reductions does not show that such reductions do not exist. The complete reductions might be deeply a posteriori or just too complex for us to specify (perhaps even infinitary).

Now for an important point emphasized by Hartry Field in his seminal "Mental Representation" (1978) and in a postscript (2001) to that essay. Philosophers often focus only on the reduction of monadic properties (with one argument place). For instance, identity theorists focus on monadic experience properties like having a headache. But, as Field notes, there also exist relations. And just as we can ask whether a monadic property is reducible, we can ask the same of a relation. To suppose the question is less pressing for relations would be an unjustified double standard.

Field focuses on relations concerning cognitive and linguistic representation: individual $x$ believes proposition $y$, individual $x$ is thinking of existing concrete object $y$, name $x$ refers to object $y$, and predicate $x$ is satisfied by object $y$. Other relations of philosophical interest include $x$ causes $y$ and fact $x$ provides a reason to perform action $y$. I have added a relation to the list: the phenomenal representation relation.

My focus is on identity theory. Identity theorists (e.g., Block, McLaughlin, Papineau) must heed Field's point. Even if they are right that monadic experience properties reduce to monadic neurocomputational properties of subjects, I have shown that they must also recognize the dyadic phenomenal representation relation between subjects and perceptible properties. And we can ask, according to identity theorists, is this 
relation reducible to some dyadic physical-functional relation between subjects and those perceptible properties, or is it not? This would require an interesting identity claim of the following form:

(1) $\lambda x \lambda y$ (subject $x$ phenomenally represents property $y)=\lambda x \lambda y(x \ldots y)$

I believe that, even if identity theorists are right that monadic experience properties are reducible to monadic neural properties, they must say that the dyadic phenomenal representation relation is irreducible. ${ }^{17}$

The strategy of my argument for this is simple. As we have seen, on internalism, BIV bears the phenomenal representation relation to roundness and other perceptible properties. But BIV is isolated from the world. So it bears no interesting dyadic physicalfunctional relation to such properties, such as the tracking relation invoked by Dretske and Tye. In brief, given internalism about experience, our standard externalist models for reducing representational relations fail in the special case of the phenomenal representation relation. I also provide a principled reason (the disjunction problem) for thinking that internalists cannot accept any alternative internalist model for reducing this relation. Let me explain these points in turn.

Broadly speaking, standard externalist theories of representational relations fall into two categories: input-based theories that emphasize what a state is apt to be caused by and behavior-oriented theories that emphasize what actions a state is apt to cause. But given internalism, both are ruled out for the phenomenal representation relation.

The tracking representationalism of Tye and Dretske discussed in section 1 provides an example of an externalist, input-based theory of phenomenal representation. Recall that tracking representationalists identify all perceptible properties, including sensible colors, with objective physical properties of external objects. Further, according to tracking representationalists, when individuals have experiences, the dyadic phenomenal representation relation that they bear to such properties is nothing but a complex tracking relation:

(2) $\lambda x \lambda y(x$ phenomenally represents property $y)=\lambda x \lambda y(x$ is in an inner state that realizes an experience and that would be caused by the instantiation of $y$ were conditions biologically normal)

Call the relation named on the right-hand side the tracking relation. Then the idea is that the phenomenal representation relation just is the tracking relation.

Let me unpack this. What makes an inner state "realize an experience"? One idea is that its content must be cognitively accessible (Tye 2000, 62; Dretske 1995, 19; Prinz 2012). Another idea is that it must satisfy only some general neural background condition (for discussion see Block 2007a). This issue does not play a role in what follows. Biologically normal conditions are conditions in which the sensory systems are "operating as they were designed to do in the sort of external environment in which they were 
designed to" (Tye 2000, 138; this is Tye's version; Dretske's is very similar). This view provides a neat account of hallucination. In hallucination, you can bear the phenomenal representation relation to properties that are not currently instantiated in your vicinity (e.g., being round and reddish), because you are in a state that would be caused by the instantiation of those properties under biologically normal conditions.

But internalists about experience cannot identify the phenomenal representation relation with the tracking relation.

To see this, consider BIV. BIV is not attached to a body. It lacks eyes and the other receptor components of the visual system. So its inner neural state, $N$, is causally cut off from the environment. It doesn't track anything under biologically normal conditions. Indeed, since BIV did not naturally evolve, there is nothing that is biologically normal for BIV.

Granted, BIV's neural state $N$ would track roundness, if $N$ were plugged into a certain situation: for instance, if it were linked with a receptor system and a body in the same way it is in normal humans (Papineau 2014, 30). But recall the basic physical facts. $N$ is just a distributed neural pattern occurring in BIV. It could have been causally connected to any shape you please (in general, anything can cause anything; the expression "round" could have been used to refer to any shape you please). For instance, if $N$ were plugged into a different neural environment, it could equally have tracked being a triangle. It also could have tracked any external color you please. In fact, if it were hooked up to a computer, it might even track the patterns of bits in that computer providing the "sensory inputs"! Since BIV lacks an evolutionary history and belongs to no species, there is nothing to select one of these counterfactual situations as the right or biologically appropriate situation for BIV. ${ }^{18}$

The conclusion that tracking theorists like Dretske and Tye would draw is that, since BIV does not track any unique set of properties (even counterfactually), it does not phenomenally represent any. So on their view, it entirely lacks experience. But, as I have argued, internalists must say that BIV does indeed have tomato-like experience $R$ and does phenomenally represent roundness and sensible redness and so on. So the conclusion they must draw is rather that the phenomenal representation is distinct from any tracking relation.

Consider next behavioral theories of representation. For instance, Evans famously said that an experience "acquires a spatial content for an organism by being linked with behavioral output" $(1985,385)$. A toy behavior-based reduction of the phenomenal representation relation might go as follows:

(3) $\lambda x \lambda y(x$ phenomenally represents property $y)=\lambda x \lambda y(x$ is in an inner state that realizes an experience and that, in typical members of the appropriate population, grounds the disposition to behave in ways "appropriate to" an object with property $y$ ) 
Call the relation named on the right-hand side the behavioral relation. Then the idea is that the phenomenal representation relation just is the behavioral relation.

So, for instance, when you (a normal human) have $N$, then you have behavioral dispositions appropriate to a red and round object at place $p$ : for instance, to reach out to place $p$ and grasp exactly as if a round thing is at $p$, and to draw a round image if asked to draw a picture of what you see, to say "that's red," and so on. And when you have an experience of a green triangle, you have another suite of sensorimotor dispositions. Such behavioral dispositions determine what properties you phenomenally represent, according to the behavioral theory.

Now, for general reasons the behavioral theory of phenomenal representation is hopeless. There is simply no road from behavior back to the content of experience. What does it even mean to say that some set of behavioral dispositions is "appropriate" to an object with property $y$ ? For instance, what are the behavioral dispositions that are uniquely "appropriate to" or "fit" an object of specific shade of red or a specific shade of white? The possibility of behaviorally undetectable spectrum inversion suggests that there is no such thing.

Even setting aside these problems, internalists about experience cannot identify the phenomenal representation relation with the behavioral relation.

To see this, consider BIV. BIV is not a complete human body. It is merely a duplicate of part of a brain. So it has no interesting behavioral dispositions. If you throw a tomato at it, it will just sit there.

Of course, in normal actual humans, $N$ grounds certain behavioral dispositions. But this point cannot save the behavioral theory. For in some other possible species, $N$ might be hooked up quite differently to a body, resulting in quite different behavioral responses. Indeed, it could be hooked up to a computer, so that its behavioral responses are digits on a computer screen. Since BIV belongs to no species and has no evolutionary history, there is nothing that could select one possible species as "the appropriate population" or one embodiment as the "normal embodiment."

It follows that BIV has no unique set of behavioral dispositions. Therefore, it does not bear the behavioral relation to any properties whatever. Nevertheless, as I have argued, internalists must say that BIV phenomenally represents roundness and sensible redness and so on. So they must conclude that the phenomenal representation relation is distinct from the behavioral relation.

In sum, BIV doesn't bear to its environment any of the causal, informational, theological, or sensorimotor relations invoked in any of our standard theories of representational relations. When it comes to providing a reductive theory of the phenomenal representation relation, it is as if internalists about experience have both hands tied behind their back. If they can provide a reductive theory of phenomenal representation, it would have to be radically different from all current theories. How might such a theory go? 
Next I consider such a reductive theory that I call "disjunctivism." But it fails. Indeed, it suggests a principled reason why any internalist reductive theory of the phenomenal representation relation must fail.

Let us focus on the brain-based identity theory of monadic experience properties. Recall that on the identity theory the tomato-like experience property $R$ is identical with the neural property $N$. Let $N 1, N 2, N 3, \ldots$ be the indefinitely-many other neural properties that, on identity theory, are identical with some experience property that individuals actually undergo (for instance, the experience of a square object, the experience of an object moving to the left, and an experience of one object above another).

We can reach "disjunctivism" about the phenomenal representation relation in two steps. First of all, if what I have argued for so far is correct, then identity theorists are committed to indefinitely-many entailments of the following form:

Having $N$ entails phenomenally representing being round (if it exists).

Having $N$ also entails phenomenally representing being reds.

Having N1 entails phenomenally representing being square.

Having N2 entails phenomenally representing moving to the left.

Having N4 entails phenomenally representing being green s. $_{\text {. }}$

Having N5 entails phenomenally representing having pitch $p$ and location $1 . .$.

The identity theorist is committed to these entailments, because each of the listed neural properties is identical with a unique experience property, which (by my argument) necessitates phenomenally representing a unique cluster of properties.

Now, this list does not specify a dyadic physical-functional relation (having two argument places) with which the dyadic phenomenal representation relation might be identified. It does not specify an identity of form (1) above.

But you might think that the list leads naturally to such an identity. For if all the above entailments obtain, perhaps identity theorists can just identify the phenomenal representation relation with some disjunctive relation of the following kind:

(4) $\lambda x \lambda y(x$ phenomenally represents property $y)=\lambda x \lambda y(x$ has neural property $N$ and $y=$ being round, or $x$ has neural property N1 and $y=$ being square, or $x$ has neural

property $\mathrm{N} 2$ and $y=$ moving to the left, or $x$ has neural property ... and $y=\ldots$ )

In short, the idea is that the phenomenal representation relation just is the disjunctive relation. This relation is basically a big list of ordered pairs. Notice this is not disjunctivism about monadic experience properties such as $R$ (we're assuming those are identical with nondisjunctive neural properties); rather, it is disjunctivism about the phenomenal representation relation.

Take an example. If you plug "BIV" into " $x$ " and "being round" into " $y$," you get a truth (because then the first disjunct becomes true). Hence BIV bears the disjunctive 
relation to being round. So on disjunctivism about phenomenal representation, BIV bears the phenomenal representation relation to being round, as desired.

On disjunctivism about phenomenal representation, nothing unifies the disjuncts. For instance, we saw that the BIV case shows that internalists cannot say that what is common between the disjuncts is that the relevant neural states track the corresponding properties.

Disjunctivism about phenomenal representation is exactly analogous to a reduction of the name-object reference relation famously considered and rejected by Field in "Tarski's Theory of Truth" (1972). On this theory, word $x$ refers to object $y$ if and only if $x$ is the word "France" and $y=$ France or $x$ is the name "Eiffel Tower" and $y=$ the Eiffel Tower, and so on for every name of English. Field notes that we would never accept such disjunctive reductions in other cases (he discusses being in pain and having valence n). So we should not accept such a theory of the reference relation.

Block $(2002,412)$ is also skeptical of such disjunctive identities because they are not "explanatory." McLaughlin is skeptical for a different reason $(2003,181)$. So these identity theorists presumably would be skeptical about disjunctivism about the phenomenal representation relation (though neither discusses this case).

Indeed, I think that identity theorists (e.g., Block, McLaughlin, Papineau) and other internalists (e.g., Chalmers, Horgan) certainly cannot accept disjunctivism about phenomenal representation for two reasons.

First, there is the modal problem. The disjunctive relation is defined in terms of a list of all the experience-constituting neural states that actual creatures undergo and the properties they actually phenomenally represent while undergoing those neural states. But surely there is a possible world where creatures have quite different neural structures and so phenomenally represent alien perceptible properties that cannot be on this list because they do not exist in the actual world. ${ }^{19}$ Hence the across-worlds extension of the phenomenal representation relation exceeds the across-worlds extension of any such disjunctive, or big-list, relation. It follows that the phenomenal representation relation is distinct from any such disjunctive relation.

There is another problem, the indeterminacy problem. I have developed the problem elsewhere (Pautz 2010a, 47-48), so here I am brief. There is actually a huge abundance of variant disjunctive relations that are candidates to be the phenomenal representation relation. They might agree in extension when it comes to actual humans but differ slightly or radically when it comes to remote actual or possible nonhumans that we never interact with. (Compare the plus and quus functions in Kripke's [1982] discussion of Wittgenstein.) For instance, suppose that in the future we come across an alien creature (perhaps an alien brain in a vat). It has a complex sensory system and undergoes a radically different kind of neural state from us. One disjunctive relation, $D$, might pair its alien brain state with perceptible property $P$. Another, $D^{*}$, might pair its alien brain state with another, radically different perceptible property, $P^{*}$. Yet another 
might pair its alien brain state with another property, $P^{* *}$. And so on. All these arbitrary disjunctive relations exist.

Now here is a problem for the disjunctivist. Intuitively, I can pretty easily refer to the phenomenal representation in my own case. And then I can go on to formulate various hypotheses about what the alien creature phenomenally represents. For instance, I might guess "the alien is aware of a shape and a color." Now the disjunctivist faces the following question: Are there any physical facts that could determine that when I make such a guess about what properties the creature is "aware of" (that is, phenomenally represents), I am determinately glomming on to one of the variant disjunctive relations of the sort described above, rather than any of the others? I think that disjunctivists must answer no. After all, all the variants fit my history of use of the predicate " $x$ is aware of $y^{\prime \prime}$ equally well. And it is not as if one of them stands out as being very natural and hence a "reference magnet" (Dorr and Hawthorne 2013). Rather, they are all equally unnatural and disjunctive. They are on a par. (In this respect, the puzzle here is unlike the puzzle about plus and quus, which might be solvable because plus is more natural than the other quus-like variants and hence a "reference magnet.") So the disjunctivist must say that it is radically indeterminate what disjunctive relation it is that $I^{\prime} m$ talking about. ${ }^{20}$ That is to say, if disjunctivism is right, then there is no determinate fact of the matter about what disjunctive relation the phenomenal representation relation is. But this has unacceptable results. For instance, it entails that I can truly say "it is indeterminate whether the alien is aware of (represents) spatial properties or whether it is aware of (represents) properties that are nothing like spatial properties" and "it is indeterminate whether the alien is aware of (represents) sensible colors or whether it instead represents properties belonging to a wholly alien quality space." Intuitively, this is absurd. Given that there is some necessary connection between phenomenology and representation, it would mean that it is radically indeterminate what it is like for the alien.

These problems undermine any possible internalist reduction of the phenomenal representation relation. For there is no general, nondisjunctive algorithm, applicable to all actual and possible individuals, going from the intrinsic characters of those individuals' neurocomputational states to the properties they phenomenally represent. So any internalist reduction of the phenomenal representation relation will inevitably identify it with a massively disjunctive relation (where there are many variant disjunctive relations in the vicinity, having different extensions). Consequently, it will be open to the problems I have identified. ${ }^{21}$ Call this cluster of issues the disjunction problem.

The conclusion I draw is that if an internalist theory of experience such as the identity theory is correct, then the phenomenal representation relation is irreducible. This view avoids all the problems I have developed for the reductive position. It may seem too radical. However, as I explain in the conclusion, it may not be as radical as it seems. 
My BIV argument for the irreducibility of the phenomenal representation relation has been entirely neutral between different solutions to the "puzzle of sensible properties" (section 2) for internalists. Indeed, some views on this strengthen my case for irreducibility. For instance, given Chalmers's general irrealist view (discussed in section 1) that in the actual world the sensible properties and even the spatial properties that we phenomenally represent are entirely uninstantiated, we can immediately rule out the claim that the phenomenal representation relation is reducible to a mind-world physical-functional relation like the tracking relation, even before we consider BIVs. Another view is Shoemaker's (1994) appearance property view, according to which sensible properties are identical with properties of the form normally causing internal neurofunctional state $F$. Shoemaker applies this view only to the sensible properties we phenomenally represent; obviously, it wouldn't be plausible to generalize it to the spatial properties we phenomenally represent. Obviously, even if this view is right, it does not absolve the internalist of the need to answer the further question, What is the dyadic phenomenal representation relation that BIV bears to such properties? My BIV argument for irreducibility of this relation applies even if Shoemaker's view is right. For instance, even if Shoemaker's view is right, the internalist cannot identify this relation with the tracking relation, for exactly the reasons I have given. Of course, the internalist might combine Chalmers's or Shoemaker's view about sensible properties with "disjunctivism" about the phenomenal representation relation. That is, he might identify the phenomenal representation relation with a disjunctive, or big-list, relation (for this theory is neutral on the metaphysical status of the perceptible properties that feature on that list). But we have already dismissed disjunctivism.

A final point about the BIV argument. The internalist about experience might accept the premise of External Directedness but still try to somehow block my BIV argument for the irreducibility of the phenomenal representation relation. But let me remind the internalist what this would require. The internalist would have to at least gesture at a general dyadic physical-functional relation, $R$, between individuals and perceptible properties (with two argument places, $x$ and $y$ ), which is a good candidate to be the dyadic phenomenal representation relation. In other words, he would have to gesture at a completion of the general schema (1). He would also have to make it plausible that this relation $R$ has the same extension as the phenomenal representation relation (e.g., that your BIV duplicate bears this relation $R$ to all the relevant perceptible properties, the same ones you phenomenally represent). Until the internalist does this, he has not provided a response. As Sider $(2011,117)$ says, if we cannot provide even a toy "metaphysical analysis" of a relation, we have excellent evidence that it is irreducible (or in a sense "fundamental" as he puts it). And the disjunction problem provides an in-principle reason for thinking that this cannot be done in the case of the phenomenal representation relation. 
We have arrived at our puzzle. If the BIV argument is sound, internalism implies that the phenomenal representation relation is irreducible. But isn't this a spooky view? Doesn't it require that internalists give up a physicalist view of the mind?

\section{Sketch of a Possible Solution: The Internal Grounding View of Phenomenal Representation}

I think that for internalists the most reasonable response to the BIV argument is to accept the conclusion that the phenomenal representation relation is irreducible. For instance, I think that accepting this conclusion is more reasonable than rejecting the premise of External Directedness, since the case for that premise is so strong. But others may not be so sure. They will regard this conclusion as extremely puzzling. So in closing I briefly sketch a view that may help reduce our sense of puzzlement. I call it the internal grounding view. I continue to focus on the brain-based identity theory, but other internalists could accept the same view. As it happens, the view meshes nicely with a general view of representational relations proposed by Paul Horwich and Stephen Schiffer on independent grounds.

First let me introduce the notion of grounding. Recently there has been a lot of enthusiasm about the explanatory potential of this notion (e.g., Fine 2001; Rosen 2010). The notion can be introduced by examples. The fact that John's action was done with the sole intention of harming grounds the fact that it is wrong. Or again, the fact that the apple is red grounds the fact that it is colored. Grounding is stronger than mere necessitation or entailment: in addition, grounding involves an explanatory or determinative connection. It also differs from reduction. For instance, as Rosen (2010) notes, followers of G. E. Moore might say that natural properties ground normative ones but deny that natural properties are reducible to normative ones. Or again, being red grounds being colored, but there is no obvious reduction in the vicinity (unless being colored is identical with a disjunction with being red as a disjunct). Many think that, at a minimum, physicalism about the mind requires mental properties and relations to be grounded in physical (and topic-neutral) ones, even if they may not be reducible to them.

Now return to the tomato-like experience property $R$. On the identity theory, $R$ is identical with neurocomputational property $N$. I have argued that identity theorists must hold that having $N$ (on this view, $R$ ) entails phenomenally representing roundness. More generally, they are committed to the raft of the neural-representational entailments gestured at in the previous section. I have also argued that they must hold that the phenomenal representation relation is irreducible.

Now for the internal grounding view. It adds only one claim: these neural states do not merely entail but also ground standing in the irreducible phenomenal representation 
relation to certain clusters of properties. This is in line with the general physicalist creed that all mental facts are grounded in physical facts.

In short, I suggest that the identity theorist must accept quite different theories for experience properties and the associated representational properties. In the case of experience properties, he can retain the reductive identity theory: they are identical with neural properties. Not so for the representational properties involved in experience. Unlike neural properties, they essentially have the form: standing in the irreducible phenomenal representation relation to spatial and other properties $P, Q, R, \ldots$ For such representational properties, the right model is grounding, not reduction. They are grounded in neural properties but not reducible to them.

Maybe this is a workable view. In fact, in a discussion of an earlier version of this chapter, Jeff Speaks endorsed its central argument, saying that "the truth of [the identity theory] would have as a surprising consequence the irreducibility of [the phenomenal representation relation]"; but he adds that "this is no immediate objection to [the identity theory]" $(2015,272)$.

For instance, the idea is that the monadic experience property $R$ is identical with the neural property $N$. This property grounds, but is not identical with, the representational property of bearing the irreducible phenomenal representation relation to roundness. Likewise, the fact that two individuals (e.g., you and your BIV duplicate) undergo the same monadic neural states grounds the fact that they bear the irreducible phenomenal representation relation to the same perceptible properties.

I conclude with some comments about this view.

(I) As I have mentioned, the internal grounding view of phenomenal representation accords nicely with a general theory of representation suggested by Horwich (1998) and Schiffer $(2003,162)$ and taken seriously by Field (2001). On this view, representational relations (believing, meaning, and similar) are generally irreducible. For instance, Schiffer writes,

What on earth could be the non-intentionally specifiable reducing relation in which "immaterial" stands to the property of being immaterial and by virtue of which the word means that property? $(2003,162)$

Nevertheless, Horwich and Schiffer hold that, whenever a thing (a word, an individual) stands in an irreducible representational relation to some item, this is grounded in (or "constituted by") its having a certain monadic physical property (a functional property, a use property, or whatever). So the view is still physicalist. As Field puts it, according to this view, "these distinct monadic properties need have nothing to do with each other, and they certainly don't need to involve a common physical relation" (2001, 71).

The internal grounding view of phenomenal representation is perfectly analogous. It concedes the irreducibility of the dyadic relation of phenomenal representation. For what on earth could be the nonintentionally specifiable relation in which BIV stands 
to the property of being round and so on, with which the phenomenal representation relation might be identified? Nevertheless, the internal grounding view holds that, whenever an individual bears the irreducible dyadic phenomenal representation relation to certain perceptible properties, this is grounded in his being in a distinct monadic neural state (which is also an experiential state).

(II) Kit Fine has suggested a congruence constraint on grounding (2001, 20-21). Fine's constraint implies that if item $P$ is real, then the ground of standing in a relation to $P$ must itself involve standing in a relation to $P$. As Fine explains, this seems generally true. For instance, typically, you refer to a thing by virtue of standing in certain underlying (e.g., informational) relations to that thing. But if the internal grounding view is true, then the congruence constraint fails when it comes to phenomenal representation, because this view holds that standing in the phenomenal representation relation to a shape property (for instance) is grounded merely in having a neural state, which is not itself a relation to that shape property. The Horwich-Schiffer view also violates Fine's principle. They conclude that the constraint is not generally valid. Horwich speaks in this connection of the "constitution fallacy" $(1998,25)$.

In fact, there may be other counterexamples to Fine's congruence constraint. For instance, the concrete, nonrelational state of an object's having a certain mass grounds the relational state of its bearing the mass-in-grams relation to a certain number. ${ }^{22}$ Also, the concrete, nonrelational state of an object's being red grounds the relational state of its instantiating the abstract universal redness (Horwich 1998, 25).

(III) The internal grounding view holds that in some cases there is a necessary connection between experience and representation. Yet it differs in several interesting ways from standard representationalist theories of experience (e.g., those defended by Dretske and Tye).

To begin with, on the internal grounding view, experience grounds phenomenal representation. For instance, on this view, the state of having the tomato-like experience $R$ is identical with the nonrelational, concrete state of being in neural property $N$. This nonrelational, concrete state then grounds the relational state of standing in the phenomenal representation relation to the abstract property being round. This fits with the key idea of the recently popular "phenomenal intentionality program" that representation is grounded in experience (e.g., Horgan 2014; Kriegel 2011; Mendelovici 2010). And it avoids the somewhat counterintuitive claim made by some representationalists that having an experience consists in standing in a representation relation to an abstract object (on the oddness of this claim see Pautz 2010c, 292ff.; and Papineau 2016, sec. 13).

The internal grounding view also provides an internalist account of phenomenal representation. Since experience is internally determined and grounds the representation of perceptible properties, the representation of perceptible properties is also internally determined. This makes phenomenal representation unique. Our standard externalist accounts (tracking accounts, teleological accounts) don't apply to it. The brain simply 
has an intrinsic capacity to phenomenally represent certain clusters of basic perceptible properties (sensible colors, shapes, and the like) that need not be instantiated in the brain.

The internal grounding view is also quite compatible with the antirepresentationalist idea that some experiential differences do not correspond to differences in the phenomenal representation of properties (Block 2007a, 538). For instance, maybe the difference between a blurry experience of a tomato and a clear one is a mere difference in the neural "content vehicle," one that doesn't ground the phenomenal representation of any different perceptible properties.

(IV) You might think that in the end the internal grounding view doesn't provide a totally satisfying solution to the puzzle of phenomenal representation. In particular, you might think it has two disadvantages.

First, the internal grounding view appears to be more complex than the kind of thoroughgoing reductive physicalism defended by philosophers like Armstrong, Field, Jackson, Lewis, Papineau, Sider, and Smart. On reductive physicalism, all properties and relations of the manifest image are just identical with complex properties and relations built from some basic stock of properties and relations from the scientific image. By contrast, the internal grounding view holds that individuals have properties of the form standing in the irreducible phenomenal representation relation to so-and-so perceptible properties that are distinct from all such complex properties, even if they are grounded in some of them. So it requires that individuals have extra properties. It also appears to require extra brute principles that a thoroughgoing reductive physicalist position would avoid. In particular, unlike reductive physicalism, this view requires grounding connections of the following kind: if an individual has complex neural property $N$, then this grounds the distinct fact that this individual phenomenally represents the property of being round. And on the internal grounding view, these appear brute. For on this view, what could possibly explain such grounding connections? On the internal grounding view, they are not derivable from a general reductive theory of the phenomenal representation relation (e.g., the disjunctivist, or big-list, view that this relation is identical with a disjunctive relation of the kind I have described previously) for the simple reason that this view rejects any such reductive theory. Despite the recent enthusiasm for grounding, brute grounding connections can be objectionable in much the same way that brute psychophysical laws or brute supervenience connections are objectionable. My point here is not just that the internal grounding view requires an explanatory gap (something all standard physicalists have learned to live with); rather, the point is that this view requires extra metaphysically brute principles, which increase the complexity of the view. ${ }^{23}$

Second, as a nonreductive view, the internal grounding view implies that there is a certain kind of nonuniformity in nature that is avoided by reductive physicalism. (David Lewis has raised a similar complaint against nonreductive views of normative properties, as discussed by Jackson $[1998,27]$.) On reductive physicalism, everywhere in nature the only properties that are instantiated are the basic stock of properties and 
relations from the scientific image, together with complex physical-functional properties $C 1, C 2, C 3, \ldots$ built up from them. But on the internal grounding view, in some cases, there is more to say. On this view, some of the complex physical-functional properties $C 1, C 2, C 3, \ldots$ are "special" in that they ground distinct properties of the form standing in the irreducible phenomenal representation relation to so-and-so perceptible properties. So, for instance, the neural property $N$ of the human brain that we have discussed is "special" in this way: it grounds the distinct property of phenomenally representing roundness. By contrast, the neural properties of the early visual system (which can be possessed in the absence of experience) do not ground any such distinct irreducible property. Likewise, the complex physical-functional properties of (say) an automobile engine do not ground any extra irreducible properties. This looks nonuniform. Why do some complex physical-functional properties in nature (viz., certain ones of the brain) ground distinct irreducible properties, while others do not?

The internal grounding view, then, appears complicated and nonuniform. However, I think it could be replied that this appearance is largely due to our lacking detailed knowledge of the brain, the most complex and amazing thing in the world. Maybe there are general, systematic grounding connections between our intrinsic neural patterns and what perceptible properties (shapes, sensible colors, and the like) we phenomenally represent, even if we have not yet discovered them (Pautz 2010b; but see Adams 1987 for interesting grounds for skepticism). And maybe, if we only knew them ("cracked the neural code"), we could look into a human brain, or BIV, and systematically decode what shapes and other perceptible properties the subject phenomenally represents. Then the internal grounding view of phenomenal representation would appear much less complicated and arbitrary. Some neuroscientists have recently worked on such brain reading (e.g., Haynes 2009). But it's still early days.

In sum, internalists face a choice. They can either try to find a fault with the BIV argument or accept a nonreductive view of the phenomenal representation relation, such as the internal grounding view. I think that their most reasonable option is to accept such a nonreductive view of the phenomenal representation relation. ${ }^{24}$ But, again, here my primary aim has been to raise a puzzle for internalists about experience-not to solve it.

\section{Notes}

1. I first developed this form of argument in Pautz (2010a, sec. 7) and Pautz (2013b). In those earlier discussions, I focused on internalism about the experience of traditional "secondary qualities" (sensible colors, audible qualities, and so on). In this chapter I focus instead on the prospects for internalism about the experience of spatial properties ("primary qualities"), a doctrine that I think raises unique puzzles. For further discussion of the puzzle I raise, and other puzzles for internalism about experience, see Speaks (2015). 
2. As we will see in section 3, External Directedness is much weaker than "representationalism," the thesis that, necessarily, all experiential facts consist in representational facts.

3. Various nonuniform theories are possible, but elsewhere (Pautz 2010b, 353-354; 2013a, 286-288) I argue that they are problematic.

4. In fact, I develop three distinct empirical arguments: the internal-dependence argument (Pautz 2010a, 2013a), the generalized structure argument (2006b, 2013a), and the judgment-explanation argument (2010b, 363n23, 358-359). Geoff Lee has suggested in discussion that there are yet other arguments against tracking representationalism based on the fact that the stimulus-signal mapping is merely probabilistic and highly context-dependent.

5. For the hypothetical coincidental variation cases, see Pautz (2006a, 2010a, 2013a). These cases not only differ from inverted spectrum and Inverted Earth cases. They also differ from Block's more recent cases of "shifted spectrum" (2007b, sec. 7) and cases of "pseudonormal vision" (2007b, sec. 10). In my view, there are problems with these cases, and they fail to undermine tracking representationalism (Pautz 2013a, 252-254; Byrne and Hilbert 2003, 19). My internal dependence argument, illustrated with coincidental variation cases, avoids these problems.

6. The recent empirical work I cite suggests that the physical ground of phenomenal similarity is neurocomputational similarity. This neural-based view of phenomenal similarity also has the advantage of allowing that across-individual comparisons of experience make sense. For instance, on the neural-based view, my color experience of a tomato might resemble a monkey's more than a dog's, if our corresponding neural patterns stand in this resemblance order. (See also Coghill, McHaffie, and Yen 2003; and Schmidt, Neitz, and Neitz 2014 on the neural ground of across-individual comparisons.) The only alternative to this neural-based view of phenomenal similarity is a behavioral-functional view according to which facts about phenomenal similarity within an individual can somehow ultimately be cashed out in terms of that individual's discriminatory and other behavioral dispositions. This view has the disadvantage of implying that across-individual comparisons of experiences never make sense (the Frege-Schlick view). True, the neural-based view requires (1) that there are well-defined measures of neural similarity and (2) that neural similarity is the causal basis of an individual's similarity judgments and ordering behavior. But I think these claims are plausible: see Pautz (2013a, 270n8) and Kriegeskorte and Kievit (2013).

7. Nevertheless, Chalmers $(2012,439)$ holds that when we say "the tomato is round" we speak truly, so there is also a sense in which his view is realist. For more on this, see note 13.

8. I have said that if Chalmers's irrealism is true, then internalism about spatial experience follows. It may be that we can also say that internalism about spatial experience is plausible only if irrealism is also true. Those who instead combine internalism with realism (e.g., Horgan 2014) face an explanatory challenge: if, contrary to externalist accounts, the brain entirely determines what properties we phenomenally represent, in a manner that is constitutively independent of links to the actual properties of external objects, then how come many of these represented propertiesnamely, the spatial properties and relations we phenomenally represent-happen to coincide with the actual properties of objects, as the realist says? Is this just some kind of lucky coincidence? Of 
course, an irrealist internalist like Chalmers avoids this explanatory challenge because he denies that there is such a coincidence to be explained: on his quite radical Edenic view, none of the properties we phenomenally represent is really out there. But for the realist internalist like Horgan (2014) there is a real question here. Elsewhere (Pautz 2011a, 418) I briefly offer a solution on behalf of the realist internalist, but I think it faces various problems, and more needs to be said.

9. For other empirical arguments against the externalist view that the experience of spatial properties can be explained in terms of tracking objective properties of objects, see Pautz $(2011 b, 394)$ and Masrour (2015).

10. Block $(2007 a, 498)$ even suggests that experience may be present in an individual (like the patient he discusses identified as GK) in the total absence of cognitive accessibility. But many find this counterintuitive; even if the concept of phenomenal consciousness is not reductively analyzable in terms of cognitive accessibility, many think that there is some necessary connection here. Nevertheless, I think that there is a potential argument for Block's suggestion. Roughly, the argument is based on a "naturalness-plus-use" theory of reference, together with the conjecture that the most natural or simple general physical property (call it $P$ ) that more or less fits our use of "experience" across individuals does not include the physical-functional machinery for cognitive access, so that it can be present without cognitive accessibility. In that case, considerations of naturalness or simplicity might support the claim that "experience" refers to physical property $P$ (and hence supports the identity "experience = physical property $P^{\prime \prime}$ ), even if this violates our pretheoretical intuition or opinion that there can be no experience in the absence of cognitive accessibility (compare Weatherson 2003 and Sider 2011, 32). An analogy is that the most natural candidate that fits our use of "water" is simply $\mathrm{H}_{2} \mathrm{O}$. A consequence is that water is in the air, even though we cannot detect it there (it is inaccessible) by ordinary methods and so are pretheoretically disinclined to say it is there. However, I think that my BIV argument is neutral on whether experience is separable from cognitive accessibility. If you think experience does require cognitive accessibility, then you can assume that BIV has the neural machinery underlying cognitive access.

11. In fact, Tye $(2009,196)$ even suggests that he has a priori justification for thinking that, if the physical facts were as described in the BIV scenario, then experience would be absent-in which case internalist theories could be swiftly ruled out from the armchair. I disagree with Tye's claim, because it goes against the generally accepted point that there are generally no a priori links between nonexperiential, physical conditions and experiential conditions (positive or negative). This general point means that, contrary to many arguments in the philosophy of mind, you cannot describe a case (e.g., the BIV case or the China-body system case) in purely physical, nonexperiential terms and then insist we have an a priori ("intuitive") justification in favor of thinking that experience is present or absent in that case.

12. For these options, see Tye $(2000,79)$ and Lycan $(1996,158)$. They advocate different versions of the idea that visual experiences have two levels of spatial content.

13. Still, Chalmers holds that when here on Earth I say "tomatoes are round" and when my twin on Twin Earth says "tomatoes are round" (speaking of the physically different tomatoes Twin Earthlings perceive), we both speak truly-our statements are "imperfectly veridical" (2006, 107; 2012, 331, 439). The reason is that Chalmers thinks the occurrences "round" in our mouths do 
not refer to the uninstantiated "perfect" roundness we both phenomenally represent (roundnessas-we-see-it) in having our identical experiences of the tomatoes. Rather, my Earthling term "roundness" refers to the quantum mechanical "imperfect roundness" instantiated by our Earthly tomatoes, whereas my twin's term "roundness" refers to the different quantum mechanical property instantiated by tomatoes on Twin Earth. (It follows that, on Chalmers's view, the ordinary English term "round" is Twin-Earthable.)

14. Nevertheless, in comments on an earlier version of this chapter, David Papineau (who accepts some form of identity theory) said that his favored response to the BIV argument I'm now developing for the irreducibility of phenomenal representation is to deny my initial premise of External Directedness, despite its strong pretheoretical pull. (Formerly, he had been more ambivalent about External Directedness-see Papineau 2014, 30.) However, he has recently suggested a replacement claim, which we might call "Internal Directedness": Necessarily, if an individual (e.g., BIV) has tomatolike experience $R$ (that is, on his identity theory, the intrinsic neural-computational property $N$ ), then there is an internal "sensory item" in the individual's experience that has a certain "visual shape," namely, roundness*, where, he says, roundness* is "an intrinsic feature of the experience" (Papineau 2016, sec. 15). By a "sensory item" Papineau must mean something like a population of neurons (or microchips or whatever) within the individual's head (as a physicalist, he cannot say that it is a nonphysical sense-datum). And roundness* must be something like a neurocomputational property, $P$, of this population of neurons. So Internal Directedness just amounts to the claim that, necessarily, if you have the tomato-like experience $R$, then there is an item in your head with a certain neurocomputational property $P$ (which Papineau somewhat misleadingly calls "roundness*," even though it is nothing like roundness). In my view, this falls well short of accommodating what is immediately plausible on reflection. What is immediately plausible on reflection is External Directedness: necessarily, if one has the tomato-like experience $R$, then one has an experience as of a round item of some kind, which matches the world only if some item is present that is round-that is, has edges roughly equidistant from a common point. Evidently, the property of being round, unlike the neurocomputational property $P$, need not be instantiated by one's neural state when one has the tomato-like experience $R$. If External Directedness is false, as Papineau thinks, then why do we all agree that BIV's experience is nonveridical, that is, fails to match its environment?

15. You might think that internalists like Mendelovici and Kriegel who reject uninstantiated properties must reject my initial claim of External Directedness, which says that the tomatolike experience $R$ is necessarily directed at a round thing. For consider a different version of the BIV case in which the property being round is not instantiated in BIV's environment and hence (according to their anti-Platonic view of properties) does not exist in that scenario. You might think that $R$ cannot be directed at a round thing in this case, if there are no round things and there is not even such a property as the property of being round. But this is not right. In fact, Mendelovici and Kriegel would accept that even in this case $R$ is necessarily directed at a round thing. For they accept a nonrelational view of the intentionality of experience on which it is totally neutral with respect to the existence of perceptible properties as well as individuals. (See also Crane [2014]. However, Crane is mostly concerned to deny that experience is fundamentally a relation to propositions; he doesn't address the issue of whether it necessarily involves being related to perceptible properties.) Now, as I explain in the text, even if they are right that having $R$ doesn't necessarily involve standing in the phenomenal representation relation to the property 
of being round, the second step of my argument ("phenomenal representation") goes through, for External Directedness still implies standing in this relation to the property of being round in the cases like the BIV case where that property exists (see also note 17). However, for the record, I myself reject this view; I think that having $R$ necessarily involves standing in the phenomenal representation relation to the property of being round, even in scenarios where nothing has this property. This implies the possibility of uninstantiated properties. For an argument for this view, and against the Kriegel-Mendelovici view, see Pautz (2007, 525-526) and Tye (2014, 51-52).

16. The conception of reduction requires a somewhat rich ontology of complex properties. As formulated, it also requires that necessary coextension is insufficient for property identity (for otherwise every property would count as reducible)_but an emendation might make it compatible with an intensionalist theory of properties (King 1998, 175n22). Other, less ontologically loaded conceptions of reduction are available: for instance, a conception invoking Sider's (2011) notion of "metaphysical semantics" or one invoking the notion of a "real definition" (to be $F$ is to be $G$ ).

17. Previously, I mentioned the anti-Platonic view of Kriegel and Mendelovici that there are no uninstantiated properties. If an internalist goes further and accepts an extreme form of nominalism on which there are only individuals and no properties at all (not even instantiated ones), then he will deny that External Directedness implies that BIV bears a phenomenal representation to clusters of perceptible properties in any scenario, for the simple reason that he thinks that there are no such things as properties. So he will dodge the question of how to reduce such a relation to a dyadic physical relation. However, given External Directedness, even the internalist who is a total nominalist must at least allow that BIV stands in a mind-world relation to concrete objects and scenes. For instance, if there happens to be a round tomato before BIV, then BIV stands in the following relation to it: $\lambda x \lambda y$ ( $x$ has an experience that is accurate with respect to object or scene $y$ ). We might call this the "veridical representation relation" (a term suggested to me by Uriah Kriegel). The arguments I employ below to argue that internalism implies the irreducibility of the phenomenal representation relation could be used, mutatis mutandis, to establish the irreducibility of this veridical representation relation. (For instance, an internalist could not provide a "disjunctive" reduction of this relation, for reasons I explain below.) So given External Directedness, even the internalist who is total nominalist cannot avoid irreducible representational relations.

18. In response to this problem of fixing the "appropriate" population for determining phenomenal representation, the internalist tracking theorist might simply specify in his account that actual humans are always the "appropriate" population. That is, he might say that $x$ (in this case, BIV) phenomenally represents property $y$ (in this case, being round) if and only if $x$ is in an internal state (in this case, $N$ ) that, in normal humans in @, tracks the instantiation of $y$. This might be called the human-centered tracking theory. But as a general account this view is a nonstarter, for several reasons. For one thing, it's absurdly chauvinistic to suppose that what perceptible properties any creature (BIV, pigeon, or whatever) phenomenally represents is a matter of what perceptible properties its inner states track in actual humans rather than some other population. Among other things, this makes facts about phenomenal representation totally arbitrary and insignificant. For instance, we can equally say that BIV "phenomenally represents" nothing but some bizarre properties involving patterns of bits, if we choose a possible population of 
brains-hooked-up-to-computers, rather than actual humans, as the "appropriate" population. For another thing, when an individual phenomenally represents a property, then she can predicate that property of things in thought because she phenomenally represents it. But it would be bizarre to suppose that BIV, which occupies a different "possible world," can predicate being round of things in thought because BIV is in a state that tracks the instantiation of roundness in humans and actualworld@ (a species and a world that are totally remote from BIV). Finally, the humancentered tracking account has the mistaken implication that, if it turns out to be the case that we are all brains in vats (i.e., it is an illusion that there is such a natural kind as humans that we belong to), then the statement "We sometimes have experiences as of round things" is falsesince in that case it is false that we are in states that track round things in actual humans. There are yet other problems of a more technical nature with the kind of actuality-based maneuver employed by the "human-centered tracking theory" (Soames 1998, 15).

19. These properties do exist in the actual world if a version of Platonism about properties is true on which, necessarily, every property is such that necessarily it exists. But needless to say, a cost of this solution to the modal problem would be a hyperabundant ontology of uninstantiated properties.

20. It might be thought that the disjunctivist can say I achieve determinate reference to one of these disjunctive relations to the exclusion of all of the others because I think of it by means of the description, the one that is the phenomenal representation relation. But this response is obviously totally wrongheaded. For one thing, it presupposes what the disjunctivist needs to explain (namely, our ability to think of the phenomenal representation relation). For another, on disjunctivism, the phenomenal representation relation just is one of the disjunctive relations, which means that disjunctivists cannot sensibly accept this "by virtue of" claim.

21. There is yet a third problem with disjunctivism. Consider again all the distinct, but very similar, disjunctive relations I gestured at previously, whose extensions agree for humans but whose extensions diverge for nonhuman creatures. Since they are very similar to one another, it would be arbitrary to suppose that one of them, to the exclusion of all the others, has a special explanatory significance in enabling us to think about certain properties and in providing immediate justification to our perceptual and introspective beliefs. Why that one? So disjunctivism is in tension with the common idea (mentioned in section 3) that phenomenal representation has a unique explanatory and epistemic significance. Hawthorne (2006, 108-109) and Lee (2013) discuss a similar issue. However, their point is that "significance" is in tension with unnaturalness, whereas my point is only that it is implausible to suppose that a relation $R$ but not a relation $R^{*}$ has explanatory significance if $R$ and $R^{*}$ are objectively very similar (a principle that is entirely neutral on their levels of naturalness).

22. There is a difference between the case of measurement and the case of phenomenal representation. While the mass-in-grams relation admits of a reduction in measurement-theoretic terms, the phenomenal representation relation cannot likewise be reduced. See Field $(2001,69-72)$ for the same point about representational relations more generally.

23. Declan Smithies pointed out to me that proponents of the internal grounding view might reply that there is a kind of explanation of the neural property $N$ always grounding the distinct 
property of standing in the irreducible phenomenally representation relation to being round. The explanation is simply that it lies in the essence of the neural property $N$ that it always grounds the distinct property of standing in the irreducible phenomenally representation relation to being round. This fits with the idea that all modal facts derive from essence (see Rosen 2010 for discussion). However, in my view, this nonreductive view doesn't make any real progress in reducing complexity, because it simply replaces brute grounding connections with brute essentialist connections between distinct properties. In fact, it is no less complicated than the initial internal grounding view. It also has the drawback of nonuniformity: it requires "special" essentialist connections concerning phenomenal representation of a kind we do not encounter elsewhere in nature. As I explain in the text's next paragraph, nonuniformity is a drawback of any nonreductive view.

24. I should say that, while I think that certain internalists (viz., identity theorists) ought to accept the internal grounding view of phenomenal representation that I have described, I do not myself accept all elements of that view. (For one thing, I am skeptical about physicalism.) But I do accept a nonreductive, internalist view of phenomenal representation along broadly the same lines (Pautz 2010a, 2010b). David Chalmers (2006, 83-84; 2012, 342-344) accepts such a view as well, although his arguments are more a priori than mine (Pautz 2013b).

\section{References}

Adams, R. 1987. Flavors, colors, and God. In The Virtue of Faith and Other Essays in Philosophical Theology, 243-262. Oxford: Oxford University Press.

Berkeley, G. 1713. First dialogue between Hylas and Philonous. In Berkeley's Philosophical Writings, ed. D. M. Armstrong. New York: Macmillan.

Block, N. 1994. Qualia. In A Companion to the Philosophy of Mind, ed. S. Guttenplan, 514-520. Oxford: Blackwell.

Block, N. 1995. On a confusion about the function of consciousness. Behavioral and Brain Sciences 18: 227-287.

Block, N. 2002. The harder problem of consciousness. Journal of Philosophy 99: 391-425.

Block, N. 2005. Two neural correlates of consciousness. Trends in Cognitive Science 9: 46-52.

Block, N. 2007a. Consciousness, accessibility, and the mesh between psychology and neuroscience. Behavioral and Brain Sciences 30: 481-548.

Block, N. 2007b. Wittgenstein and qualia. Philosophical Perspectives 21: 73-115.

Block, N. 2009. Comparing the major theories of consciousness. In The Cognitive Neurosciences, 4th ed., ed. M. Gazzaniga, 1111-1122. Cambridge, MA: MIT Press.

Block, N., and J. Fodor. 1972. What psychological states are not. Philosophical Review 81: 159-181.

Block, N., and K. O'Regan. 2012. Discussion of J. Kevin O'Regan's Why Red Doesn't Sound Like a Bell: Understanding the Feel of Consciousness. Review of Philosophy and Psychology 3: 89-108. 
Brouwer, G., and D. Heeger. 2009. Decoding and reconstructing color from responses in human visual cortex. Journal of Neuroscience 29: 13992-14003.

Brouwer, G., and D. Heeger. 2013. Categorical clustering of the neural representation of color. Journal of Neuroscience 33: 15454-15465.

Burge, T. 2010. Origins of Objectivity. Oxford: Oxford University Press.

Byrne, A., and D. Hilbert. 2003. Color realism and color science. Behavioral and Brain Sciences 26: 3-21.

Chalmers, D. 2004. The representational character of experience. In The Future for Philosophy, ed. B. Leiter, 153-182. Oxford: Oxford University Press.

Chalmers, D. 2006. Perception and the fall from Eden. In Perceptual Experience, ed. T. Gendler and J. Hawthorne, 49-125. Oxford: Oxford University Press.

Chalmers, D. 2012. Constructing the World. Oxford: Oxford University Press.

Chang E. F., J. W. Rieger, K. Johnson, M. S. Berger, N. M. Barbaro, et al. 2010. Categorical speech representation in human superior temporal gyrus. Nature Neuroscience 13: 1428-1432.

Coghill, R., J. McHaffie, and Y. Yen. 2003. Neural correlates of interindividual differences in the subjective experience of pain. Proceedings of the National Academy of Sciences 100: 8538-8542.

Crane, T. 2014. Aspects of Psychologism. Cambridge, MA: Harvard University Press.

Crouzet, S. M., N. A. Busch, and K. Ohla. 2015. Taste quality decoding parallels taste sensations. Current Biology 25: 1-7.

Danilova, M., and J. Mollon. 2012. Cardinal axes are not independent in color discrimination. Journal of the Optical Society of America 29: 157-164.

Dorr, C., and J. Hawthorne. 2013. Naturalness. Oxford Studies in Metaphysics 8: 3-77.

Dretske, F. 1995. Naturalizing the Mind. Cambridge, MA: MIT Press.

Evans, G. 1985. Molyneux's question. In The Collected Papers of Gareth Evans, 364-399. London: Oxford University Press.

Field, H. 1972. Tarski's theory of truth. Journal of Philosophy 13: 347-375.

Field, H. 1978. Mental representation. Erkenntnis 13: 9-61.

Field, H. 2001. Postscript to "mental representation." In Truth and the Absence of Fact, 68-82. Oxford: Oxford University Press.

Fine, K. 2001. The question of realism. Philosopher's Imprint 1: 1-30.

Hawthorne, J. 2006. Postscript: Sider's Argument from Vagueness. In Metaphysical Essays, 104-109. Oxford: Oxford University Press.

Haynes, J. 2009. Decoding visual consciousness from human brain signals. Trends in Cognitive Science 13: 194-202. 
Horgan, T. 2014. Phenomenal intentionality and secondary qualities: The quixotic case of color. In Does Perception Have Content? ed. B. Brogaard, 329-350. Oxford: Oxford University Press.

Horwich, P. 1998. Meaning. Oxford: Oxford University Press.

Howard, J. D., J. Plailly, M. Grueschow, J. D. Haynes, and J. A. Gottfried. 2009. Odor quality coding and categorization in human posterior piriform cortex. Nature Neuroscience 12: 932-939.

Jackson, F. 1998. From Metaphysics to Ethics. Oxford: Oxford University Press.

King, J. 1998. What is a philosophical analysis? Philosophical Studies 90: 155-179.

Kriegel, U. 2011. The Sources of Intentionality. Oxford: Oxford University Press.

Kriegeskorte, N., and R. A. Kievit. 2013. Representational geometry: Integrating cognition, computation, and the brain. Trends in Cognitive Sciences 17: 401-412.

Kripke, S. 1982. Wittgenstein on Rules and Private Language. Cambridge, MA: Harvard University Press.

Lee, G. 2013. Materialism and the epistemic significance of consciousness. In Current Controversies in the Philosophy of Mind, ed. U. Kriegel, 222-245. London: Routledge.

Levine, J. 2001. Purple Haze. Oxford: Oxford University Press.

Lycan, W. 1996. Consciousness and Experience. Cambridge, MA: MIT Press.

Masrour, F. 2015. The geometry of visual space and the nature of visual experience. Philosophical Studies 172: 1813-1832.

McLaughlin, B. 2003. A naturalist-phenomenal realist response to Block's harder problem. Philosophical Issues 13: 163-204.

Mendelovici, A. 2010. Mental representation and closely conflated topics. PhD diss., Princeton University.

Ney, A. 2013. Ontological reduction and the wave function ontology. In The Wave Function: Essays in the Metaphysics of Quantum Mechanics, ed. A. Ney and D. Alber, 168-183. Oxford: Oxford University Press.

Papineau, D. 2014. I-The presidential address: Sensory experience and representational properties. Proceedings of the Aristotelian Society Hardback 114: 1-33.

Papineau, D. 2016. Against pepresentationalism (about conscious sensory experience). International Journal of Philosophical Studies 24: 324-347.

Pautz, A. 2006a. Sensory awareness is not a wide physical relation: An empirical argument against externalist intentionalism. Noûs 40: 205-240.

Pautz, A. 2006b. Can the physicalist explain colour structure in terms of colour experience? Australasian Journal of Philosophy 84: 535-565.

Pautz, A. 2007. Intentionalism and perceptual presence. Philosophical Perspectives 21: 495-541. 
Pautz, A. 2010a. A simple view of consciousness. In The Waning of Materialism, ed. G. Bealer and R. Koons, 25-66. Oxford: Oxford University Press.

Pautz, A. 2010b. Do theories of consciousness rest on a mistake? Philosophical Issues 20: 333-367.

Pautz, A. 2010c. Why explain experience in terms of content? In Perceiving the World, ed. B. Nanay, 254-309. Oxford: Oxford University Press.

Pautz, A. 2011a. Can disjunctivists explain our access to the sensible world? Philosophical Issues 21: $384-433$.

Pautz, A. 2011b. Review of Hill's Consciousness. Analysis Reviews 71: 393-397.

Pautz, A. 2013a. The real trouble with phenomenal externalism: New empirical evidence for a brain-based theory of consciousness. In Consciousness Inside and Out: Phenomenology, Neuroscience, and the Nature of Experience, ed. R. Brown, 237-298. New York: Springer.

Pautz, A. 2013b. The real trouble with armchair arguments against phenomenal externalism. In New Waves in Philosophy of Mind, ed. M. Sprevak and J. Kallestrup, 153-181. London: Palgrave.

Peacocke, C. 2008. Sensational properties: Theses to accept and theses to reject. Revue Internationale de Philosophie 62: 7-24.

Prinz, J. 2012. The Conscious Brain. Oxford: Oxford University Press.

Rosen, G. 2010. Metaphysical dependence: Grounding and reduction. In Modality: Metaphysics, Logic and Epistemology, ed. B. Hale and A. Hoffman, 109-136. Oxford: Oxford University Press.

Schiffer, S. 2003. The Things We Mean. Oxford: Oxford University Press.

Schmidt, B., M. Neitz, and J. Neitz. 2014. Neurobiological hypothesis of color appearance and hue perception. Journal of the Optical Society of America 31: 195-207.

Shoemaker, S. 1994. Phenomenal character. Noûs 28: 21-38.

Sider, T. 2011. Writing the Book of the World. Oxford: Oxford University Press.

Soames, S. 1998. The modal argument: Wide scope and rigidified descriptions. Noûs 32: 1-28.

Speaks, J. 2015. The Phenomenal and the Representational. Oxford: Oxford University Press.

Tye, M. 1995. Ten Problems of Consciousness. Cambridge, MA: MIT Press.

Tye, M. 2000. Consciousness, Color, and Content. Cambridge, MA: MIT Press.

Tye, M. 2009. Consciousness Revisited. Cambridge, MA: MIT Press.

Tye, M. 2014. Transparency, qualia realism and representationalism. Philosophical Studies 170: 39-57.

Weatherson, B. 2003. What good are counterexamples? Philosophical Studies 115: 1-31.

Youngentob, S. L., B. A. Johnson, M. Leon, P. R. Sheehe, and P. F. Kent. 2006. Predicting odorant quality perceptions from multidimensional scaling of olfactory bulb glomerular activity patterns. Behavioral Neuroscience 120: 1337-1345. 